\title{
Magnetospheric Convection in the Nondipolar Magnetic Field of Uranus
}

\author{
RICHARD S. SELESNICK ${ }^{1}$
}

\author{
Department of Physics and Center for Space Research, Massachusetts Institute of Technology, Cambridge
}

\begin{abstract}
A method for determining the magnetospheric convection electric field using simple analytic approximations under the assumption of uniform ionospheric conductivity is described, and applied to Uranus. Magnetic field models including quadrupole and octupole moments are used to determine the shape of the polar caps and the mapping of the electric field and parallel currents between ionosphere and magnetosphere. The asymmetry in the magnetic field models between the northem and southern hemispheres leads to the inclusion of currents between the hemispheres in order to satisfy the assumption of equipotential magnetic field lines. The results show that the quadrupole moment of the Uranian magnetic field strongly influences magnetospheric convection, and that a significant octupole moment will further alter the flow pattern. Even with these modifications the basic flow is sunward in the inner magnetosphere as inferred previously. The total current which flows along field lines between the two hemispheres due to the asymmetry of the magnetic field is comparable in magnitude to that of the region 1 current system. Time dependent calculations including a self-consistent electric field show that ring current shielding of the electric field is important and may have formed the most prominent features in the plasma observations made by Voyager 2. The effectiveness of the shielding can be influenced by the magnetic field model. Other features in the data are characteristic of substorm injection, and the model has been used to show that a combination of plasma injection and electric field shielding may be applicable to the interpretation of the Voyager 2 data.
\end{abstract}

\section{INTRODUCTION}

One of the most surprising results from the Voyager 2 encounter with Uranus in January, 1986, was the detection of a magnetic field whose dipole moment is tilted from the planetary rotation axis by an angle of about $60^{\circ}$ [Ness et al., 1986] and which contains a strong quadrupole component [Connerney et al., 1987]. The tilt of the magnetic field and the orientation of the planetary rotation axis quickly led to the realization that, contrary to preencounter expectations, the Uranian magnetosphere is not rotation dominated like those of Jupiter and Saturn. Instead, as in the Earth's magnetosphere, plasma transport is controlled by solar wind-driven convection [Vasyliunas, 1986; Selesnick and Richardson, 1986; Selesnick, 1987]. This conclusion is based on simple assumptions about the direction of the convection electric field. In this paper the convection electric field is calculated in more detail by considering the effects introduced by magnetosphere-ionosphere coupling and the nondipolar aspects of the planetary magnetic field.

At present, Uranus is at the point in its 84 year orbit where the rotation axis is pointing almost directly at the Sun. Due to the tilt of the dipole axis, the magnetic field at any given time is fairly similar in orientation with respect to the solar wind to that of Earth and the other planets. However, the field at a fixed point constantly changes due to the rotation of the planet. Therefore the direction of the convection electric field, which depends on the directions of both the planetary magnetic field and solar wind, changes with time, but is approximately constant in the corotating reference frame. Equipotentials of the convection electric field are also plasma streamlines, but the plasma only moves along its streamlines if they are constant in time. Therefore the plasma flow is determined by the equipotentials in the corotating reference frame and is not influenced by the corotation electric

${ }^{1}$ Now at California Institute of Technology, Pasadena

Copyright 1988 by the American Geophysical Union.

Paper number 8 A9565.

0148-0227/88/008A-9565\$05.00 field which is zero in this frame. By transforming the flow back to an inertial reference frame the plasma fluid elements are found to follow helical trajectories in the sunward direction, leading to the conclusion that solar wind-driven convection transports plasma through the magnetosphere even in the presence of a strong corotation electric field [Vasyliunas, 1986; Selesnick and Richardson, 1986; Selesnick, 1987].

It has also been shown [Selesnick and Richardson, 1986; Selesnick, 1987] that the slight time dependence of the solar wind direction in the corotating reference frame, introduced by the small angle $\left(\sim 8^{\circ}\right)$ between the rotation axis and the planet-Sun line, does not theoretically introduce any significant effects. The magnetosphere will start to become rotation dominated only when Uranus has progressed significantly around its orbit of the Sun. Therefore for the present location of Uranus, equipotentials in the corotating reference frame provide a good approximation to the plasma trajectories in that frame.

The numerical model which is described herein is based on the convection model of the Earth's magnetosphere which has been developed over the last several years at Rice University (see Harel et al. [1981] and the review by Wolf [1983]). The primary physical differences between the models for the two planets are that we do not include a corotation electric field and use magnetic field models which are relevant to Uranus. Also several simplifying assumptions are made in the present study. The primary assumption is that the ionospheric conductivity of Uranus is uniform, allowing an analytic approximation to the electrostatic potential.

The magnetic field models for Uranus are described in the next section, together with a discussion of their influence in determining the shapes of the polar caps. Then a brief review of the equations governing magnetosphere-ionosphere coupling is given. The calculation of the convection electric field is divided into two parts. First we calculate the field due to the influence of the solar wind in determining the potential at the polar cap boundaries, which we call the external field, and second, the field due to the drift of hot plasma in the magnetosphere, which we call the self-consistent field. Finally, comparisons of the model predictions with plasma data taken by Voyager 2 in the inner 
Uranian magnetosphere are discussed, followed by a summary of results.

\section{MAGNETIC FIEID AND POLAR CAP MODELS}

From measurements taken by the Voyager 2 magnetometer, Connerney et al. [1987] have constructed a spherical harmonic expansion of the Uranian magnetic field. Because of the limited spatial extent of the measurements, they were reliably able to constrain only the expansion coefficients up to and including the quadrupole terms. The octupole part of the field was not determined. However, in order to obtain the best possible estimate of the quadrupole coefficients, octupole coefficients were also included in the fit to the data, as well as a uniform external field due to currents outside the modeling region. The resulting field model, consisting of the dipole and quadrupole terms only, is called the $\mathrm{Q}_{3}$ model and provides the most accurate description of the Uranian magnetic field available from the Voyager 2 data. However, as discussed by Connerney et al. [1987], the necessity for including octupole terms in the fit indicates that such terms are, in fact, present in the planetary field, and it is a result of the Voyager 2 trajectory that reliable estimates of them were not obtainable from the data. The unusually large quadrupole and octupole moments are thought to be due to part of the field being generated in Uranus' ice mantle which surrounds the core [Dolginov, 1986; Connerney et al., 1987].

In this study three magnetic field models have been used. They are each derived from the spherical harmonic expansion of Connerney et al. [1987] by including successively the dipole, quadrupole and octupole coefficients. The ratios of the total dipole, quadrupole and octupole moments in the models are $1: 0.70: 0.43$. The second model, consisting of dipole and quadrupole terms, is the $Q_{3}$ model. The first model with dipole terms only differs from the offset-tilted-dipole (OTD) model of Ness et al. [1986] because it is centered on the planet. The direction of the dipole moment, however, is similar to that of the OTD model. The model which includes the octupole terms is not expected to produce a reliable determination of the true planetary field, but is included to estimate the importance of any effects caused by the octupole component. The dipole model is included for comparison with an earthlike magnetic field. The three field models are illustrated in Figure 1. Here the position of Voyager 2 is shown at 15 minute intervals from 1500 to 2000 SCET (spacecraft event time), in a coordinate system in which the $Z$ axis (vertical), is along the planetary dipole vector and the $R$ axis (horizontal), is the cylindrical distance from the dipole axis. At each point the magnetic field line which instantaneously threads the spacecraft position is plotted for the particular field model. In the context of this study, the polar caps are defined as the regions poleward of the boundaries separating antisunward from sunward ionospheric convection in each hemisphere. Since the magnetic field models do not include the contributions of magnetopause and magnetotail currents, the polar cap boundaries are determined by mapping a circle of radius $20 R_{U}\left(1 R_{U} \equiv 25,600 \mathrm{~km}\right)$ centered on the planet from the equatorial plane, along magnetic field lines, to the northern and southern ionosphere (at $1 R_{U}$ ). Here, and in what follows, we define the equatorial plane as the extension of the magnetic equator in the dipole field model. The value of $20 R_{U}$ was chosen because the resulting polar caps contain a magnetic flux of about $5 \times 10^{9} \mathrm{~Wb}$ which is approximately the amount of magnetic flux in each lobe of the magnetotail of Uranus [Behannon et al., 1987]. The resulting polar cap boundaries can be thought of as the boundaries between open and closed field lines for merging of the planetary field with an

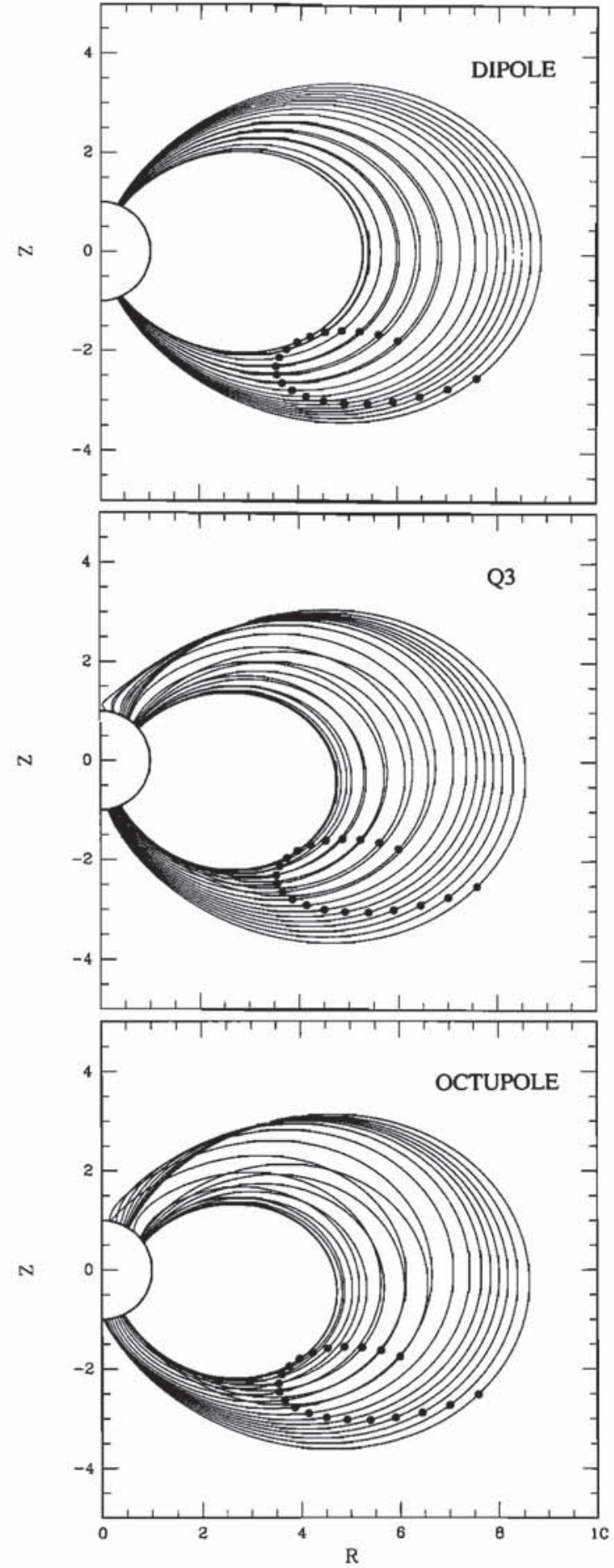

Fig. 1. Field lines for the dipole, $Q_{3}$, and octupole magnetic field models described in the text. The points show positions of Voyager 2 at 15 minute intervals from 1500 to 2000 SCET on January 24,1986 . The $Z$ axis is along the planetary dipole moment and the $R$ axis is cylindrical distance.

interplanetary magnetic field which is directed parallel to the dipole moment of the planet, ignoring the effect of the solar wind in forming a magnetotail. For the dipole model the polar cap boundaries are the intersection of the ionosphere with $L=20$ and are therefore circular and centered on the magnetic poles. Since the polar caps of the Earth are approximately circular, the polar caps given by the above prescription should provide an adequate representation of their true shapes. The technique for determining 
the polar cap boundaries is also useful for comparison between the three magnetic field models because at a radial distance of $20 R_{U}$ the field of each model is approximately dipolar.

\section{MAGNETOSPHERE-IONOSPHERE COUPLING}

The evolution of the electrostatic potential in the ionosphere is controlled by the coupled set of equations [e.g., Wolf, 1983]:

$$
\begin{gathered}
\nabla \cdot \Sigma \cdot \nabla \Phi=j_{\|} \sin \psi \\
j_{\|}=\frac{1}{2} \frac{B_{i}}{B_{e}}\left(\nabla E_{k} \times \hat{\mathbf{b}}_{e}\right) \cdot \nabla \eta \\
\frac{\partial \eta}{\partial t}+\mathbf{v}_{d} \cdot \nabla \eta=0 \\
\mathbf{v}_{d}=\frac{\hat{b}_{e} \times \nabla\left(E_{k}+q \Phi\right)}{q B_{e}} \\
\Sigma=\left[\begin{array}{ll}
\frac{\Sigma_{P}}{\sin ^{2} \psi} & \frac{\Sigma_{H}}{\sin \psi} \\
\frac{-\Sigma_{H}}{\sin _{k}} & \Sigma_{P}
\end{array}\right]
\end{gathered}
$$

\section{The notation is}

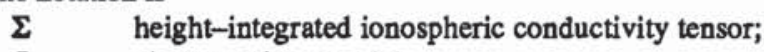

$\Phi$ electrostatic potential;

$j_{\|} \quad$ current density parallel to the magnetic field;

$\psi \quad$ magnetic dip angle at the ionosphere;

$B_{i} \quad$ magnetic field magnitude at the ionosphere;

$B_{c} \quad$ magnetic field magnitude at the equatorial plane;

$E_{k} \quad$ particle kinetic energy;

$\hat{b}_{e}$ unit vector along magnetic field in the equatorial plane;

$\eta \quad$ flux tube particle content ( $\int n d s / B$ where $n$ is number density and $d s$ is along the magnetic field);

$\mathbf{v}_{d} \quad$ bounce-averaged gradient/curvature plus $\mathbf{E} \times \mathbf{B}$ drift velocity;

$q \quad$ charge on plasma species;

$\lambda$ adiabatic invariant for isotropic plasma;

$V \quad$ flux tube volume ( $\left.\int d s / B\right)$;

$\Sigma_{P} \quad$ height-integrated ionospheric Pedersen conductivity;

$\Sigma_{H} \quad$ height-integrated ionospheric Hall conductivity.

Here it has been assumed that the magnetic field lines are equipotentials, the plasma in the magnetosphere remains isotropic through pitch angle scattering, there are no sources or losses of magnetospheric plasma in the region of interest, there are no ionospheric neutral winds, and the magnetic field is symmetrical between the northern and southern hemispheres. The last assumption will be relaxed for the $\mathrm{Q}_{3}$ and octupole magnetic field models. The ionosphere has been idealized as a conducting spherical shell characterized by the height-integrated Pedersen and Hall conductivities. Equation (6) is expressed in a coordinate system with the axis corresponding to the first diagonal element along the projection of the local magnetic field onto the ionosphere.

Equation (1) expresses conservation of charge in the ionosphere by relating the divergence of the ionospheric surface current $(=-\Sigma \cdot \nabla \Phi$ by Ohm's law) to the current entering or leaving the ionosphere along field lines. Equation (2) expresses conservation of charge in the magnetosphere. The parallel current leaving a magnetospheric flux tube has been derived from the divergence of the ring current due to bounce-averaged gradient and curvature drifts for particles of a given kinetic energy. Equation (3) expresses the conservation of particles in a drifting flux tube. Equation (4) is the drift velocity of the flux tube for particles of a given kinetic energy. Equation (5) is the adiabatic compression law for particles confined to the flux tube volume. The assumption of isotropic particle distributions allows the magnetosphere to be described by flux tube-averaged properties such as $\mathbf{v}_{d}$, which are then given as functions of position, $\mathbf{r}_{e}$, on a two-dimensional surface, normally taken to be the equatorial plane. If this surface is not the equatorial plane then $B_{e}$ in (2) and (4) should be changed accordingly. The particle population of a given flux tube is described in (3) and (5) by two constants, $\eta$ and $\lambda$, which are conserved by the motion of the flux tube. It is assumed that only one particle species is present, that is, one value of $\lambda$ with corresponding $\eta\left(\mathbf{r}_{e}\right)$, but the equations can easily be generalized to many populations by including the appropriate summation in (2).

If the conductivities $\Sigma_{P}$ and $\Sigma_{H}$ are uniform and the magnetic field is perpendicular to the ionosphere then $\Sigma$ becomes the identity matrix times $\Sigma_{P}$ and (1) becomes

$$
\nabla^{2} \Phi=\frac{j_{\|}}{\Sigma_{p}}
$$

The two-dimensional Laplacian in (7) may be transformed from spherical coordinates $(\theta, \phi)$, to polar coordinates $(R, \phi)$, by the substitution

$$
R=\left(\tan \frac{\theta}{2}\right)^{ \pm 1}
$$

where the plus and minus are for the northern and southern hemispheres, respectively, and $\theta$ is the dipole magnetic colatitude. Equation (8) transforms each hemisphere to a unit disc with the dipole axis at the center and the equator at the circumference. Solutions to Laplace's equation on a disc are well known, so (7) represents a useful approximation to (1) and is adopted throughout. The validity of the approximation is discussed in the following section.

We solve (7) in two steps. First we assume that there are no parallel currents from the magnetosphere except at the polar cap boundary where appropriate boundary conditions are applied. The resulting solution is termed the external field. The solution with parallel currents from the magnetosphere equatorward of, but not at, the polar cap boundary is termed the self-consistent field, which can be superimposed on the external field. Since the self-consistent field depends on assumptions about the magnetospheric plasma distribution, it is useful to consider the external field separately.

\section{EXTERNAL FIEID}

The effect of antisunward ionospheric convection inside the polar caps is simulated by a boundary condition on the electrostatic potential at the polar cap boundaries. Since the open field lines within the polar caps are expected to be influenced by the solar wind or cross-tail electric fields at large radial distances, the boundary condition is chosen at the $20 R_{U}$ circle in the equatorial plane, which defines the polar caps, and is then mapped along field lines to the ionosphere. For simplicity, the potential is chosen to vary sinusoidally around the circular equatorial boundary, following Vasyliunas [1970], in a sense which provides antisunward flow within the polar caps. Again, because of the similarity in the field models at large radial distance, a ready 
comparison of the effects of the different magnetic fields on high-latitude convection is available. The postulated boundary condition on the potential can alternatively be considered as the application of an idealized region 1 current system which flows on a current sheet intersecting the polar cap boundary, with the region 2 currents (to be discussed in the next section) determining the self-consistent field.

In the absence of $j_{\|}$, (7) reduces to Laplace's equation. To solve Laplace's equation equatorward of the polar cap boundary, a low latitude boundary condition on the potential is also required. We choose to have the latitudinal gradient of the potential be zero at the equator. If Hall currents are neglected then this condition implies no current flow across the equator, which is the required condition for the dipole field model. As discussed below, and by Harel et al. [1981], the solution is relatively insensitive to the equatorial boundary condition, so the one adopted here should be adequate. Because the approximation of the magnetic field being perpendicular to the ionosphere only holds at high latitudes and an equatorial electrojet is not included in the model, the solutions are not expected to be valid near the equator in any case. For the $\mathrm{Q}_{3}$ and octupole models the magnetic dip equator is substantially different than the dipole magnetic equator (the $\mathrm{Q}_{3}$ model actually has a separate dip equator in the northern hemisphere). The solutions will therefore not be valid in these regions and again the equatorial boundary condition becomes less valid. However, we are primarily interested in the potential which maps to regions in the equatorial plane at distances greater than about $3 R_{U}$ from the planet, so these effects should not be significant.

With the boundary conditions just described, the ionospheric potential outside the polar caps for the dipole field model is easily found to be

$$
\Phi_{e x t}=\Phi_{p}\left[\frac{R+1 / R}{R_{p}+1 / R_{p}}\right] \sin \phi
$$

where the subscript ext refers to the external field, $\Phi_{p}$ is half of the total potential drop across the polar caps and $R_{p}$ is the radius of the polar caps in the $(R, \phi)$ plane. A similar solution can also be found inside the polar caps. Potential contours for the solution (9) are shown in Figure 2 in the $(R, \phi)$ plane and projected along magnetic field lines into the equatorial plane.

For the dipole field model, with uniform ionospheric conductivities, the ionospheric Ohm's law (1) can be reduced to a differential equation in $R$ alone which is easily integrated numerically. Similar techniques were used in the early convection models [Fejer, 1953, 1964; Vasyliunas, 1970, 1972]. Potential contours, projected into the equatorial plane, resulting from the numerical integration with no ionospheric Hall conductivity $\left(\Sigma_{H}=0\right.$ in (6)) are shown in Figure 3. Comparing with the analytic approximation shown in Figure 2, which was obtained by assuming that the magnetic field is perpendicular to the ionosphere, shows that the approximation is valid outside $L \approx 3$. Since the equatorial boundary condition is not strictly valid, such accuracy should be sufficient.

For the $\mathrm{Q}_{3}$ and octupole magnetic field models the azimuthal component of the magnetic field leads to an azimuthal dependence in the conductivity tensor because (6) must then be rotated so that the diagonal elements correspond to the polar and azimuthal directions. Therefore (1) cannot be reduced to a one-dimensional equation even with uniform conductivity. To solve (1) exactly would require a numerical integration in two-dimensions such as that used in the Rice convection model [Harel et al., 1981]. We shall adopt the approximation used in
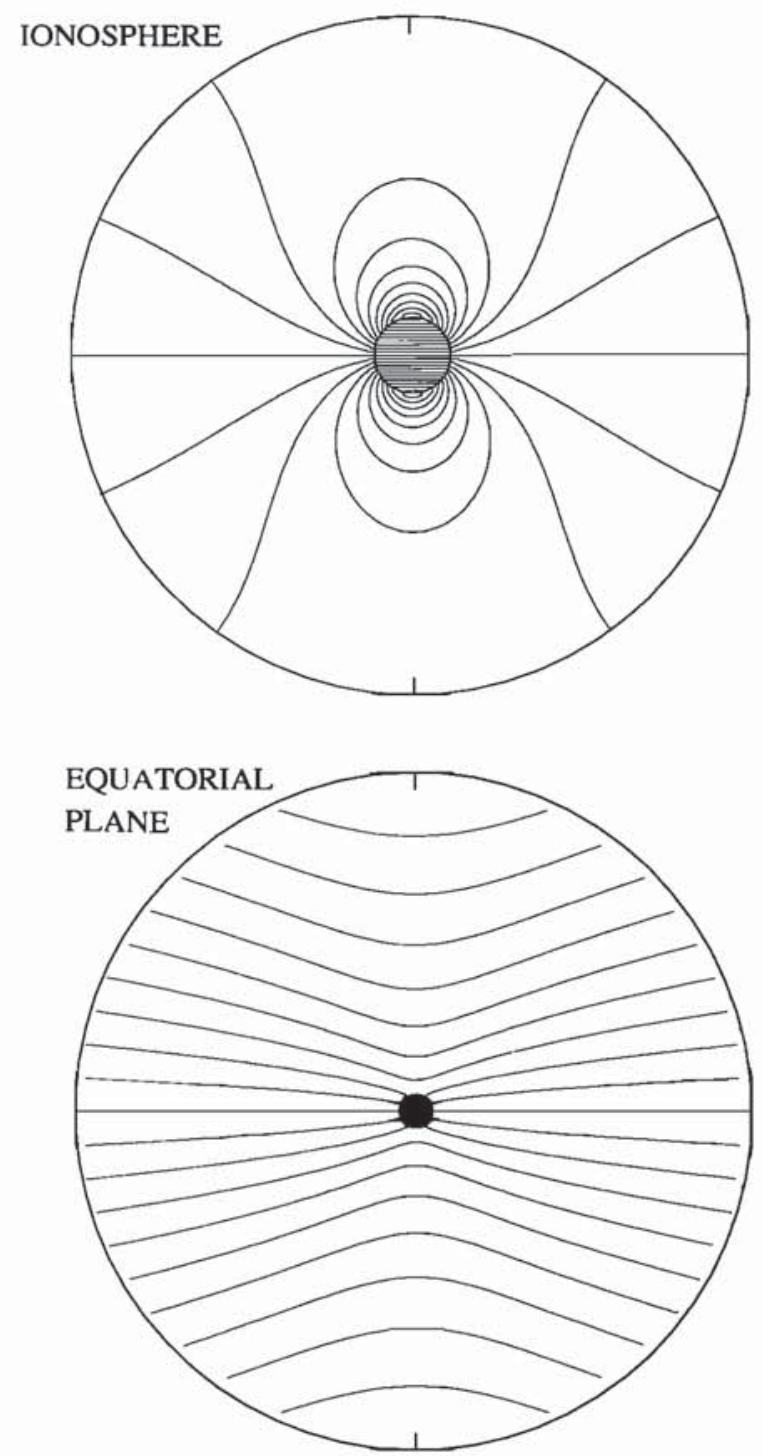

Fig. 2. (top) Equipotentials of the analytic approximation to the external field for the dipole model in the ionospheric $(R, \phi)$ coordinates. The inner circle is the polar cap boundary with anti-sunward convection inside. The outer circle is the equator at $R=1$. (bottom) The same solution mapped along magnetic field lines into the equatorial plane. The solid inner circle now represents the planet and the outer circle is $20 R_{U}$.

(T) which provides sufficient accuracy away from the equator. The solution (9) cannot be applied directly, however, because the polar caps are not circular or centered on the magnetic poles. In order to apply (9) the polar cap boundaries are first transformed to a centered circle by conformal mapping. This is done in two parts. First, the polar cap boundary is mapped to a circle by the inverse of a Joukowski transformation [e.g., Saff and Snider, 1976, p.361]:

$$
w=z+\frac{C_{1}}{z^{2}}
$$

which is often used in aerodynamics to map circles to airfoils. By choosing an appropriate value for the constant $C_{1}$, the circle can be distorted into a shape which is close to the polar cap shapes obtained from both the $Q_{3}$ and octupole magnetic field models. Next the circle is shifted to be centered on the origin while maintaining the equator at $R=1$ using a bilinear transformation: 


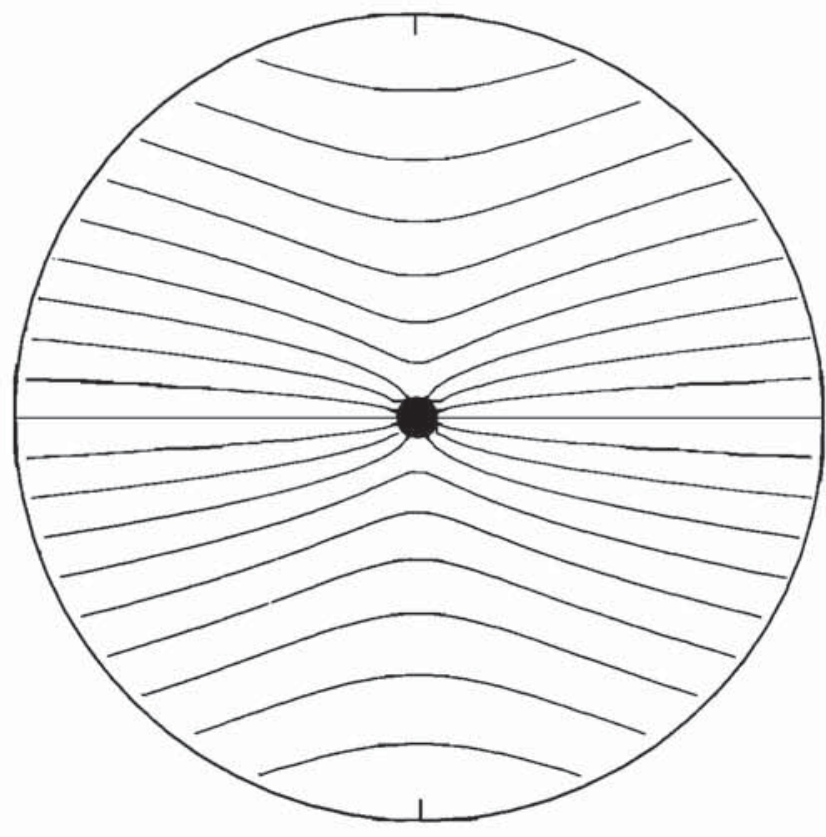

Fig. 3. Equipotentials in the equatorial plane of the exact external field for the dipole model with uniform ionospheric conductivity, obtained by numerical integration.

$$
w=\frac{C_{2}-C_{3} z}{C_{2} C_{3} z-1}
$$

The complex constants $C_{1}, C_{2}$ and $C_{3}$ are determined by a least squares fit of a number of points on the polar cap boundaries to points on the final circle. Good fits were obtained by choosing 50 evenly spaced points. The conformal mapping causes the polar boundary condition on $\Phi$, when applied in the $(R, \phi)$ plane, to depart from the simple sinusoidal form which is valid in the equatorial plane. However, (9) is easily extended to include higher harmonics, and by expanding the boundary condition in a Fourier series the solution to Laplace's equation is obtained. In practice only the first two or three terms of the Fourier expansion make a significant contribution to the final potential. The results of the above procedure are shown in Figure 4 for the $Q_{3}$ model and Figure 5 for the octupole model. Figures 4 and 5 show the polar cap boundary and potential contours in the $(R, \phi)$ plane for the northern magnetic hemisphere at the top, and similar plots for the southern magnetic hemisphere at the bottom. The conformal mapping does not apply inside the polar caps so the solutions there are not shown. In these and subsequent figures the same contour levels are plotted for comparison and can be scaled to the total cross-polar potential drop, $2 \Phi_{p}$.

Figure 6 shows the potential contours for the $Q_{3}$ model projected into the equatorial plane from both the northern and southern magnetic hemispheres. Different potential contours are obtained from each hemisphere, even though the boundary potentials (at $20 R_{V}$ ) are the same. The difference is a result of asymmetry in the magnetic field between the two hemispheres and cannot be removed by adjusting the boundary conditions at the two polar caps. It was assumed in mapping the potential from the ionosphere to the equatorial plane that the magnetic field lines are equipotentials. However, the solutions shown which exhibit different potentials at each end of a given field line violate this assumption. In order to reinstate the condition of equipotential field lines, parallel currents must flow along the potential gradient between the hemispheres. These currents will leave the ionosphere at one end of the field line and enter it at the other end, modifying the potential according to (1) until the potentials at both ends of every field line are equal. The currents then close in the ionosphere across the magnetic equator. For the $Q_{3}$ and octupole magnetic field models the problem of finding the external field has now become more difficult because the parallel currents, $j_{\|}$, on the right-hand side of (7) are not zero but must be determined simultaneously with the potential, $\Phi$.

We first consider the solution to (7) if $j_{\|}$is a known function. For non-zero parallel currents (7) can be solved by use of a Green's function:

$$
\Phi(\mathbf{R})=\int G\left(\mathbf{R}, \mathbf{R}^{\prime}\right) j_{\mathbb{N}}(\mathbf{R}) d \mathbf{R}^{\prime}
$$

The form of the Green's function, $G(R, R)$, depends on the boundary conditions. If we assume that $G$ is zero on the polar cap
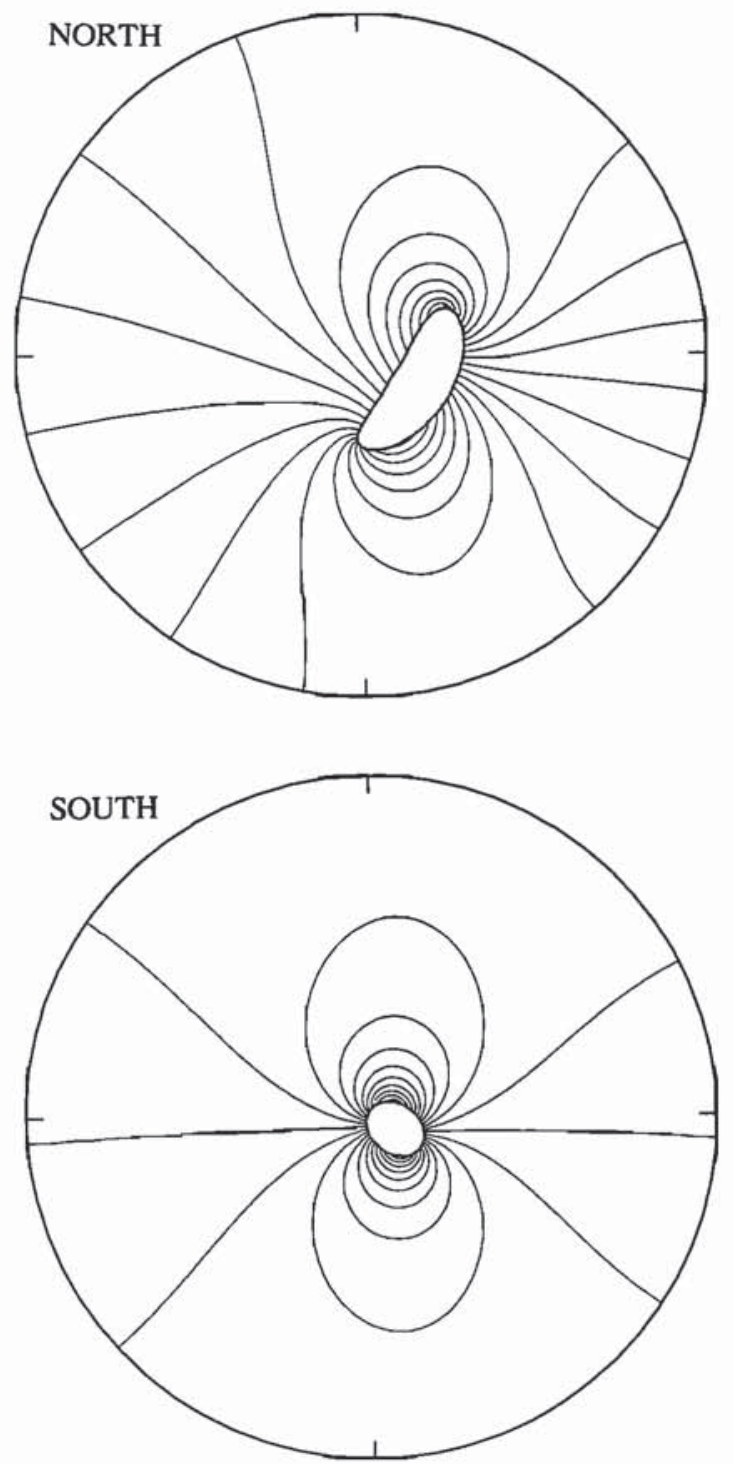

Fig. 4. Equipotentials in the ionosphere centered on the magnetic dipole axis for the $Q_{3}$ model in the northern and southern magnetic hemispheres, using the analytic approximation. The polar cap boundaries are also shown and the Sun is to the left. 

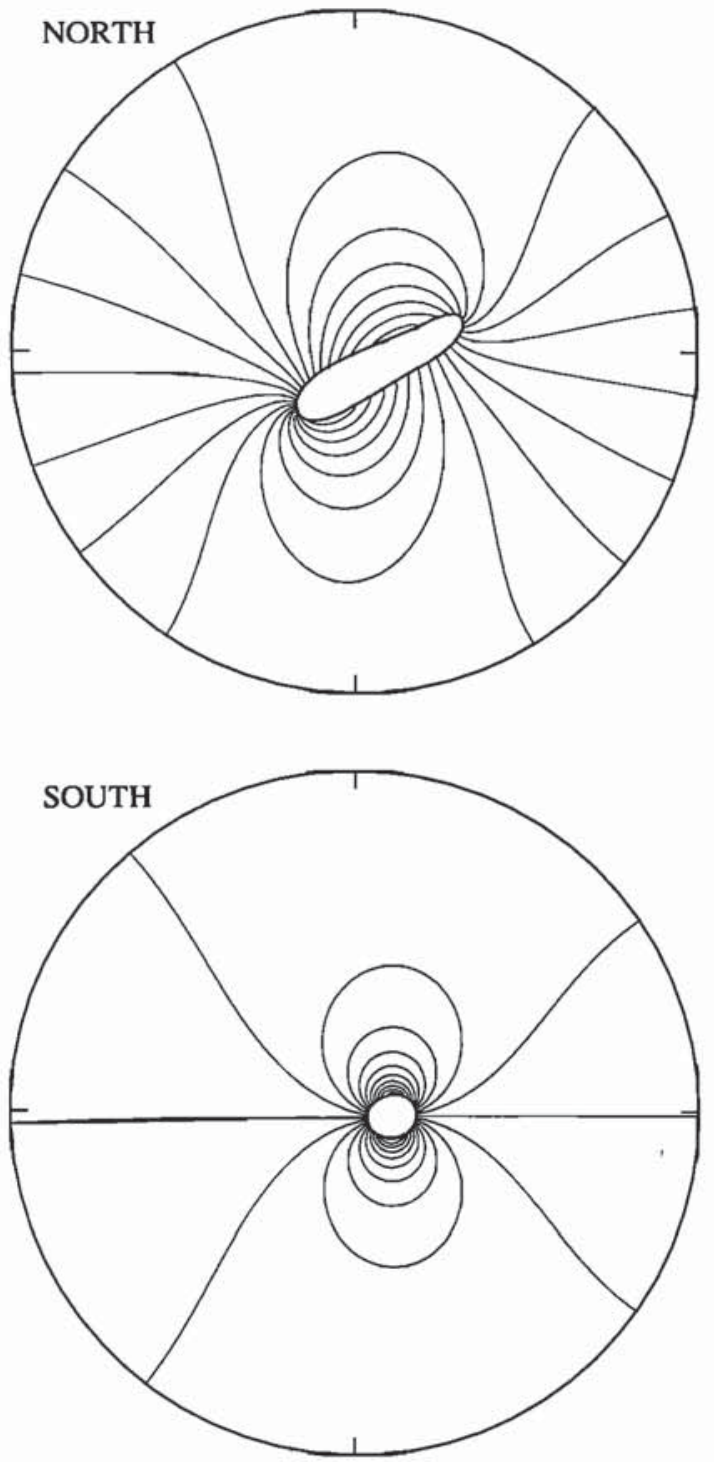

Fig. 5. Same as Figure 4 for the octupole model.

boundary and satisfies the same equatorial boundary condition (zero latitudinal gradient) as the external field, then the solution (12) may be added to the previous solution with $j_{\|}=0$ to obtain the full external field. In this case $G$ can be found by the method of images (see Morse and Feshbach [1953, pp. 1240-1242] for the derivation of a similar Green's function) and is given by

$$
\begin{aligned}
G\left(\mathbf{R}, \mathbf{R}^{\prime}\right)= & \frac{\sin ^{2} \theta}{2 \pi \Sigma_{p} R^{2}} \operatorname{Re} \log \left[\cot \left[\frac{\pi}{2 a} \log \frac{z}{z^{\prime}}\right]+\cot \left[\frac{\pi}{a} \log \frac{R^{\prime}}{R_{p}}\right]\right] \\
& \times\left[\cot \left[\frac{\pi}{2 a} \log \frac{z R^{\prime}}{z^{\prime}}\right]+\cot \left[\frac{\pi}{a} \log \frac{1}{R R^{\prime}}\right]\right]
\end{aligned}
$$

where $z$ and $z^{\prime}$ are complex forms of $\mathbf{R}$ and $\mathbf{R}^{\prime}$, and $a=-2 \log R_{p}$. A simpler Green's function can be found by ignoring the equatorial boundary condition and is given by

$$
G\left(\mathbf{R}, \mathbf{R}^{\prime}\right)=\frac{\sin ^{2} \theta}{2 \pi \Sigma_{p} R^{2}} \log \left[\frac{R_{0}}{R^{\prime}} \frac{\left|\mathbf{R}-\mathbf{R}^{\prime}\right|}{\left|\mathbf{R}-\left(R_{0} / R^{\prime}\right) \mathbf{R}^{\prime}\right|}\right]
$$

which is valid at high latitudes. Figure 7 shows a comparison of the potential contours for the Green's functions given by (13) (top) and (14) (bottom). The effects of the different boundary conditions are illustrated. Since the approximation in (7) is valid only at high latitudes as discussed above, the Green's function given by (14) will be used. In theory it may be possible (although probably not practical) to find a Green's function for the full operator in (1) with a given conductivity model.

To find $j_{\|}$, the condition of equipotential field lines is expressed as

$$
\Phi_{0}^{N}\left(\mathbf{R}^{M}\right)+\Phi_{1}^{N}\left(\mathbf{R}^{N}\right)=\Phi_{0}^{S}\left(\mathbf{R}^{S}\right)+\Phi_{1}^{S}\left(\mathbf{R}^{S}\right)
$$

The subscripts 0 and 1 refer, respectively, to the solutions obtained with no $j_{\|}$, shown in Figures 4 and 5 , and to the solutions with $j_{\|}$given by (12). The superscripts $N$ and $S$ refer to the north and south hemispheres and $\mathbf{R}^{N}$ is the point in the northem $(R, \phi)$ plane whose field line maps to $\mathbf{R}^{s}$ in the southern $(R, \phi)$ plane. An additional constraint is that the current per unit magnetic flux is conserved along a flux tube, that is, the current
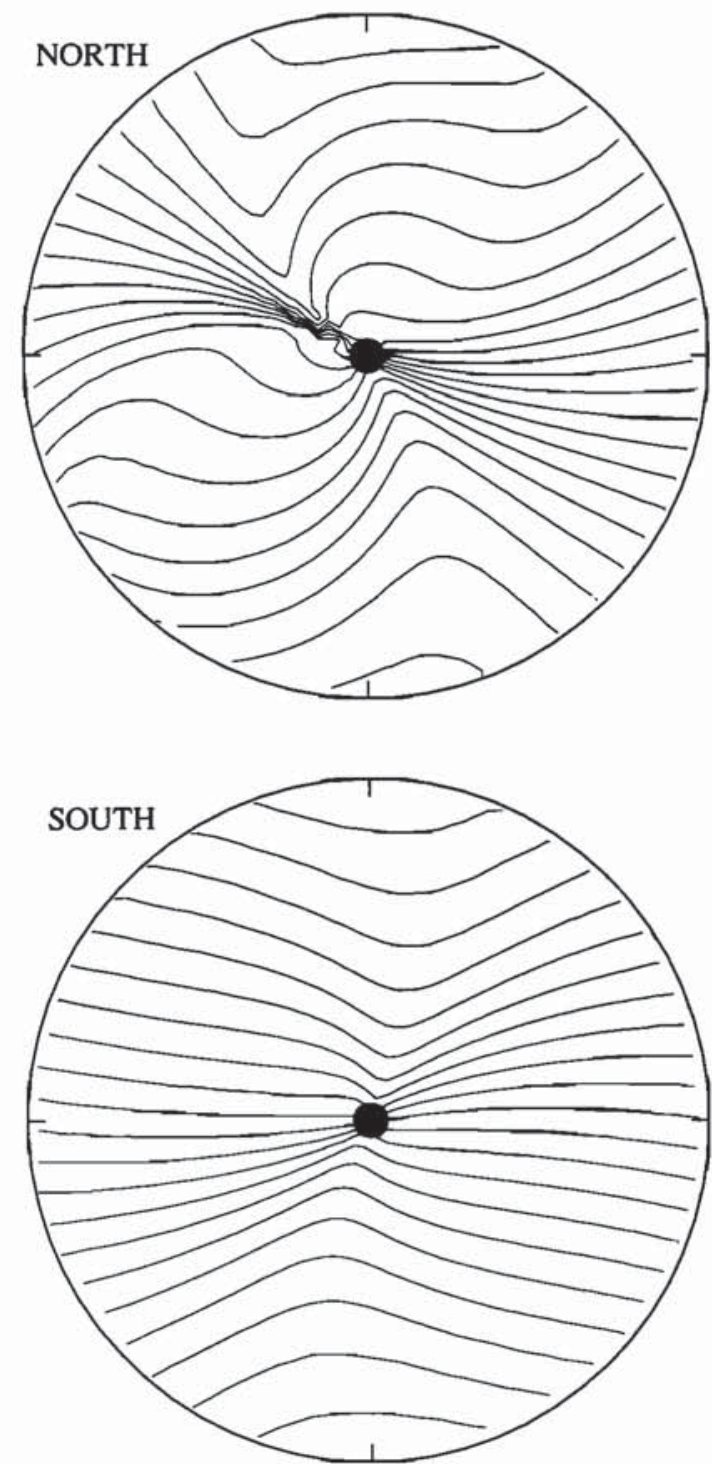

Fig. 6. Potential for the $Q_{3}$ model mapped into the equatorial plane from the northernand southem magnetic hemispheres. The Sun is to the left. 

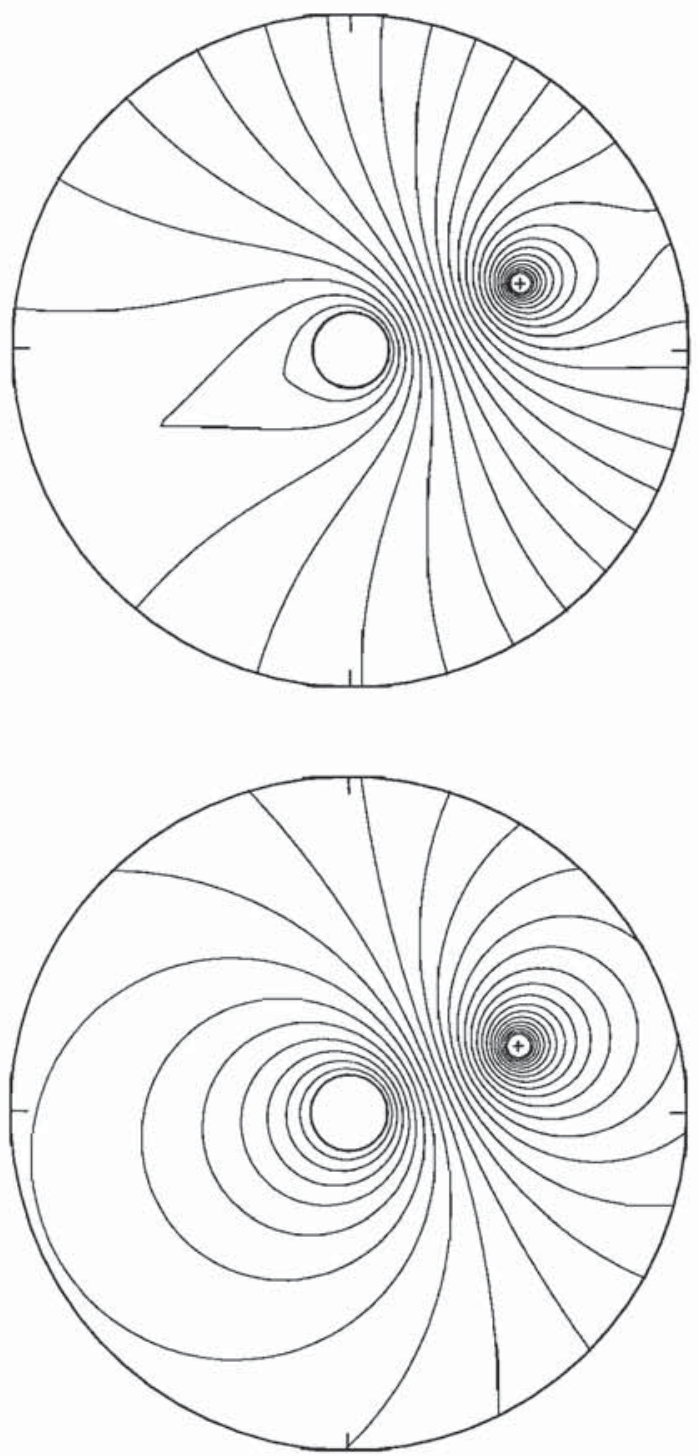

Fig. 7. Equipotentials for the two Green's function giveu by (top) (13) and (bottom) (14). The plus is the location, $\mathbf{R}^{\prime}$, of the point charge.

leaving the ionosphere from one foot of a flux tube is equal to that entering at the other foot. Therefore

$$
j_{\|}^{S}\left(\mathbf{R}^{S}\right)=-\frac{B^{S}\left(\mathbf{R}^{S}\right)}{B^{N}\left(\mathbf{R}^{N}\right)} j_{\|}^{N}\left(\mathbf{R}^{N}\right)
$$

where $B$ is the magnetic field magnitude at the ionosphere. Substituting (12) for the unknown potentials $\Phi_{1}^{N}$ and $\Phi_{1}^{S}$ in (15), and (16) for $j_{\|}^{S}$, gives an integral equation for $j_{\|}^{N}$ :

$$
\begin{gathered}
\Phi_{0}^{S}\left(\mathbf{R}^{S}\right)-\Phi_{0}^{N}\left(\mathbf{R}^{N}\right)= \\
\int\left[G\left(\mathbf{R}^{N}, \mathbf{R}^{\prime N}\right)+\frac{B^{S}\left(\mathbf{R}^{S}\right)}{B^{N}\left(\mathbf{R}^{N}\right)} J G\left(\mathbf{R}^{S}, \mathbf{R}^{\prime S}\right)\right] j_{\|}^{N}\left(\mathbf{R}^{\prime M}\right) d \mathbf{R}^{\prime N}
\end{gathered}
$$

where $J$ is the Jacobian of the transformation from $\mathbf{R}^{S}$ to $\mathbf{R}^{N}$ $\left(J=\left|\partial \mathbf{R}^{S} / \partial \mathbf{R}^{N}\right|\right)$ and $\mathbf{R}^{S}$ is implicitly a function of $\mathbf{R}^{N}$.

An integral equation like (17) can be solved numerically by converting it to a matrix equation

$$
y=A x
$$

whose solution is given by

$$
x=\left(A^{T} A+\varepsilon l\right)^{-1} A^{T} y
$$

Here $x$ and $y$ are column vectors, $A$ is a (not necessarily square) matrix, $I$ is the identity matrix and $\varepsilon$ is a damping constant introduced for numerical stability. The integral equation (17) is easily cast into the form of (18) by converting $j_{\|}$to a set of discrete currents which flow along a chosen set of field lines to form the vector $x$, and by evaluating the left-hand side at a different set of points in the ionosphere to form the vector $y$. Once the set of discrete currents has been evaluated via (19), they can be converted to potentials by a matrix form of the Green's function integral (12).

Unfortunately, the matrix method requires a prohibitively large amount of computation in order to obtain sufficient resolution in the final set of potentials. By applying (19) with a sufficiently small number of currents to make it practical (up to 64 discrete currents have been used), we can only obtain an estimate of the amount of parallel current which flows because of the magnetic field asymmetry. If the number of discrete currents in the vector $x$ is smaller than the number of potential differences in the vector $y$, then the solution given by (19) provides a set of currents which give potentials that are the closest possible, in the least squares sense, to the actual solution [e.g., Menke, 1984]. Results from such an application of (17) show that the net current between the hemispheres is small ( $\sim 5 \%)$ compared to the total region 1 current. The total current (irrespective of sign) which flows between the hemispheres increases as more discrete currents are added to the model to improve the resolution in the final solution. For the octupole model, even with the low obtainable resolution, the total current is found to be comparable in magnitude to the total region 1 current (somewhat less for the $\mathrm{Q}_{3}$ model). The size of the region 1 current is estimated from the change in the latitudinal potential gradient across the polar cap boundary and is about $2 \times 10^{6}$ A for typical values (see later discussion) of $\Phi_{p}=20 \mathrm{kV}$ and $\Sigma_{P}=4 \mathrm{mho}$.

If $G$ is approximated by replacing the logarithm in (14) by a delta function, $\delta\left(\mathbf{R}-\mathbf{R}^{\prime}\right)$, then the integral in (17) can be evaluated explicitly. The potential of a field line becomes a weighted average of the potential at each end:

$$
\Phi_{e x t}=\frac{\Phi_{0}^{N}+\alpha \Phi_{0}^{S}}{1+\alpha}
$$

where

$$
\alpha=\frac{B^{N}}{B^{S}}\left[\frac{R^{N} \sin \theta^{s}}{R^{S} \sin \theta^{N}}\right]^{2}
$$

Obviously, this is only a gross approximation because the potential of a given field line should depend on $\Phi_{0}$ from all of the other field lines, weighted by some function of their distance. However, the weighting factor $\alpha$ is essentially a ratio of the magnetic field magnitudes in each hemisphere, with the factor in parentheses being close to unity for small $\theta$ according to the transformation (8) (note that $R$ is dimensionless). Therefore the final potential is weighted toward $\Phi_{0}$ from the hemisphere with the weaker magnetic field. For the $Q_{3}$ and octupole models the magnetic field in the northern magnetic hemisphere is substantially weaker than that in the southern hemisphere, as can be seen from Figure 1 and the relative sizes of the polar caps in Figures 4 and 5 . Therefore $\Phi_{0}$ from the northern hemisphere should dominate, and in this sense (20) provides a reasonable approximation. The results of averaging the potentials shown in 


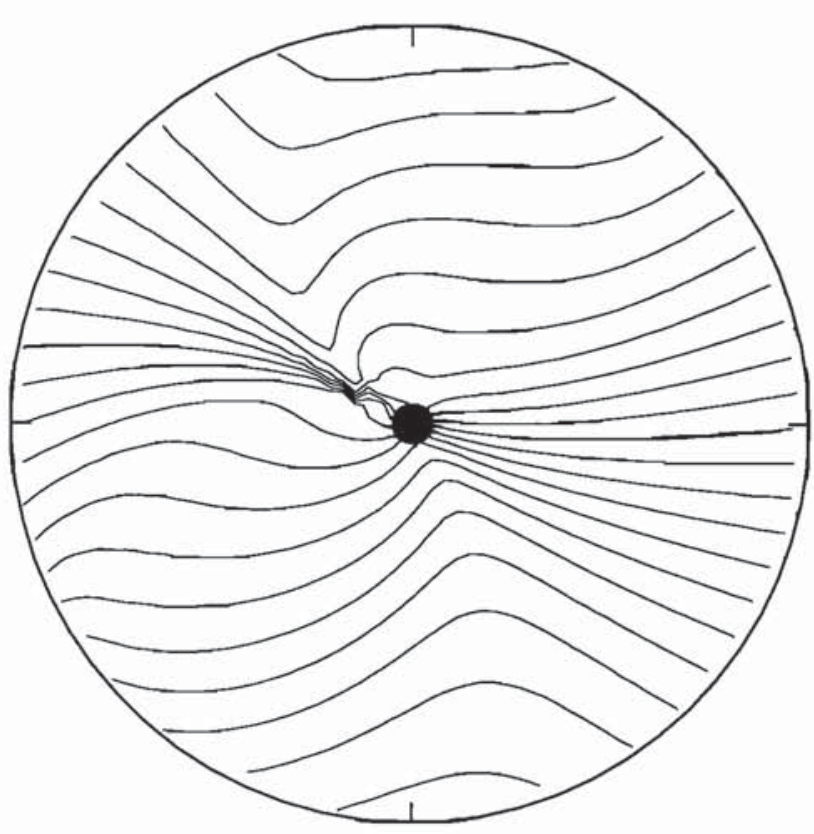

Fig. 8. Average potential, according to (16), for the $Q_{3}$ model.

Figures 4 and 5 according to (20) are shown for the equatorial plane in Figures 8 and 9 for the $Q_{3}$ and octupole models, respectively. It is seen that the final potential is indeed close to $\Phi_{0}$ from the northem hemisphere (compare Figures 6 and 8).

\section{SELF-CONSISTENT FIEID}

The method for determining the self-consistent field has already been given in the previous sections. For a given magnetospheric plasma population with an energy invariant $\lambda_{\text {, }}$ distributed in the equatorial plane according to a given density invariant $\eta\left(\mathbf{r}_{e}\right)$, parallel current is produced according to (2). With $j_{\|}$determined, the self-consistent field is given by the

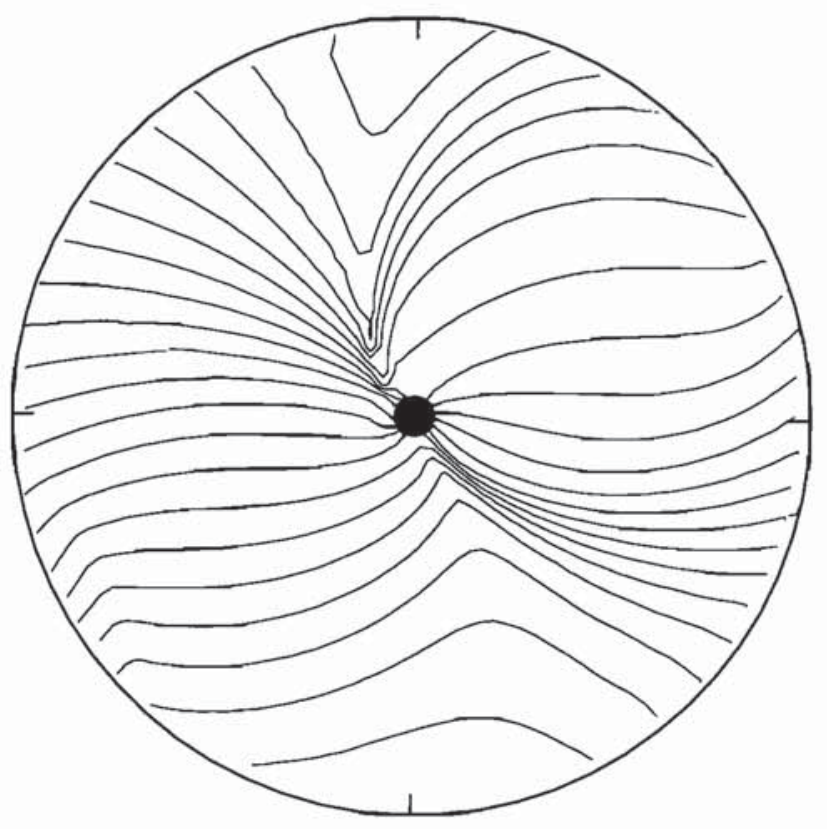

Fig. 9. Same as Figure 8 for the octupole model.
Green's function integral (12). Again, the potential of a given field line will be different in each hemisphere and needs to be averaged according to (20). For symmetric magnetic fields, such as the dipole model, $j_{\|}$from the magnetosphere is divided equally between the two ionospheric hemispheres. For the asymmetric field models $j_{\|}$is adjusted by parallel currents flowing between the hemispheres according to the integral equation (17). Therefore the initial choice of how the currents from the magnetosphere are divided is unimportant because they are corrected afterwards. However, since we are only using the approximation of averaging on a single field line according to (20), it is advantageous to minimize the change in the potential due to the averaging. This is done by modifying (2) so that the currents are divided using the weighting factor $\alpha$, which appears in (20), with less current going to the hemisphere with higher magnetic field. Such a modification is used in subsequent calculations. The division of currents using $\alpha$ is done to give approximately equal current densities in the $(R, \phi)$ plane of each hemisphere.

The potential, $\Phi$, is now determined as the sum of the external and self-consistent fields for a specified magnetospheric plasma distribution. The time evolution of the system is found by using the drift velocity (4), with a given time step, to move the particles to new positions and then recalculating $j_{\|}$to repeat the process. The resulting iterative solution is a simplified version of that used in the Rice convection model [Harel et al., 1981], except that the assumption of uniform ionospheric conductivity allows the Green's function solution to be used.

To minimize the computation in evaluating $j_{\|}, \eta$ is assumed to be uniform everywhere except across a given curve in the equatorial plane where it jumps from zero to a finite value. The curve is defined by connecting a set of points with line segments of length $\Delta l_{i}$. A discrete current is then associated with the midpoint of each line segment and, from (2), is given by

$$
I_{i}=\eta \frac{\partial E_{k}}{\partial l} \Delta l_{i} \csc \theta_{b}
$$

where the derivative is taken along the line segment, and $\theta_{b}$ is the angle between the line segment and the local magnetic field, which are not exactly perpendicular for the $Q_{3}$ and octupole models. Each of the currents $I_{i}$ is divided between the two hemispheres with the weighting factor $\alpha$ to give a set of currents entering the northern and southern $(R, \phi)$ planes. The integral over the Green's function to give the potential is then reduced to a summation in each hemisphere. The potential is evaluated at each of the original points which form the gradient in $\eta$, or plasma edge, and the drift velocity at each point is used to increment the location of the edge.

As discussed in the next section, the Voyager 2 plasma data show a sharp density gradient near $5 R_{U}$ on both the inbound and outbound legs of the encounter. Therefore, in order to simulate the plasma distribution, the convection model has been run starting with a plasma inner edge which is a circle centered on the planet. Such a plasma distribution does not produce any self-consistent field for the dipole magnetic field model because the contours of constant flux tube volume are also circles concentric with the planet, so that the kinetic energy gradient along the boundary in (21) is zero. As the model is stepped forward in time, the inner edge is convected toward the day side of the magnetosphere so that the self-consistent field gradually becomes important.

After allowing the model to run for a sufficiently long time with a constant external field, a steady state configuration is reached in which the plasma inner edge becomes stationary. 

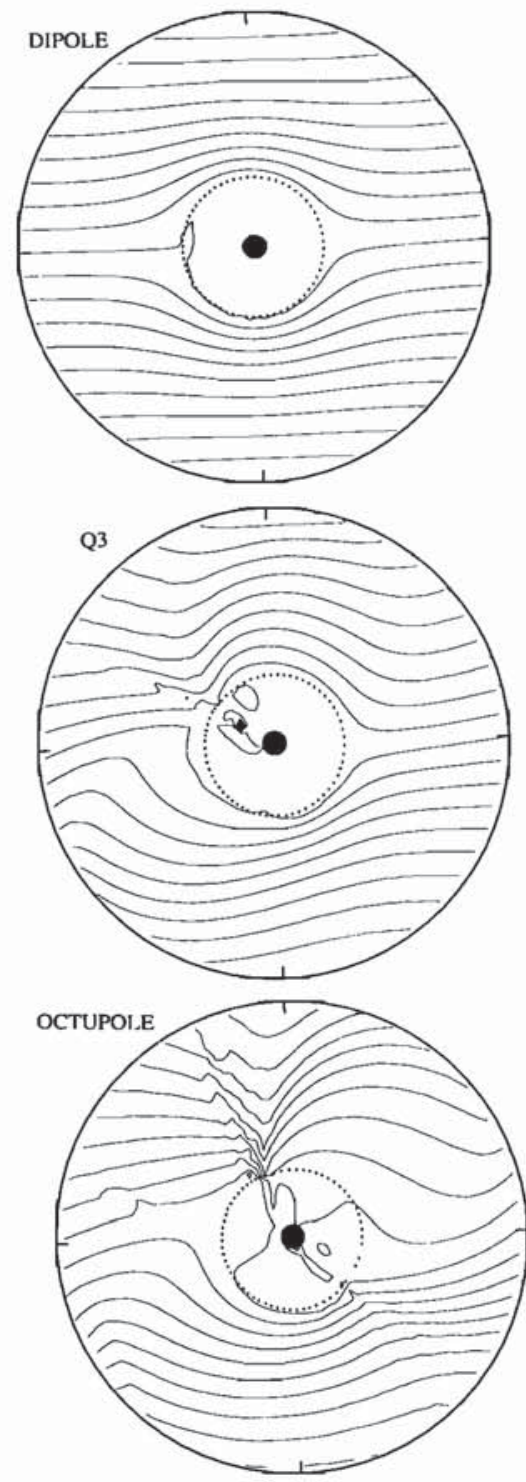

Fig. 10. Steady state solutions for the total (external plus self-consistent) field with a plasma inner edge (indicated by + signs) near $6 R_{U}$ for the dipole, $Q_{3}$, and octupole magnetic field models. The Sun is to the left.

Steady state configurations for each of the three magnetic field models are shown in Figure 10. These were reached after a 10-hour simulation (see the discussion of shielding time below) with a time step of $3 \mathrm{~min}$. The final shape and location of the inner edge are sensitive to the assumed initial configuration, to $\lambda$, $\Phi_{p}$ and the ratio $\eta q / \Sigma_{p}$ [Vasyliunas, 1972]. For the calculations shown in Figure 10, the parameters were chosen to be $\lambda=2 \mathrm{MeV}\left(R_{U} / G\right)^{2 / 3}, \Phi_{p}=20 \mathrm{kV}, \eta q / \Sigma_{P}=40$, with the inner edge, made up of 64 points, at $6 R_{U}$. The chosen $\lambda$ value corresponds to an energy of about $5 \mathrm{keV}$ at $L=6$. The justification for these values is discussed in the next section. In Figure 10, shielding of the external field from low latitudes by the self-consistent field produced at the plasma inner edge is evident. Such shielding is well documented in the convection models of the Earth's magnetosphere [Vasyliunas, 1972; Southwood, 1977; Harel et al., 1981; Senior and Blanc, 1984] and in empirical models of the convection electric field in the Earth's magnetosphere [Volland, 1973; Stern, 1977; McIlwain, 1986].
It is also of interest to know how the self-consistent field responds to changes in the external field. During a magnetic substorm, for example, the external field is subject to a rapid increase at the time of substorm onset. Such an increase is easily introduced into the model by varying the cross-polar cap potential drop, $\Phi_{p}$, during d run. Figures 11,12 and 13 show the results of suddenly changing $\Phi_{p}$ from 20 to $50 \mathrm{kV}$ after the steady state configurations of Figure 10 were reached. For each magnetic field model the potential contours and inner edge are shown for three times; immediately after the increase in $\Phi_{p}$ after 1 hour and after 6 hours. The extent of, and time scale for, reinstatement of shielding for each field model is evident. The shielding time has been discussed by several authors [Jaggi and Wolf, 1973; Southwood, 1977; Siscoe, 1982; Senior and Blanc, 1984] and depends on $\lambda$ and $\eta q / \Sigma_{p}$. The results of the present model are in agreement with the analytical derivations of the shielding time given by these authors.

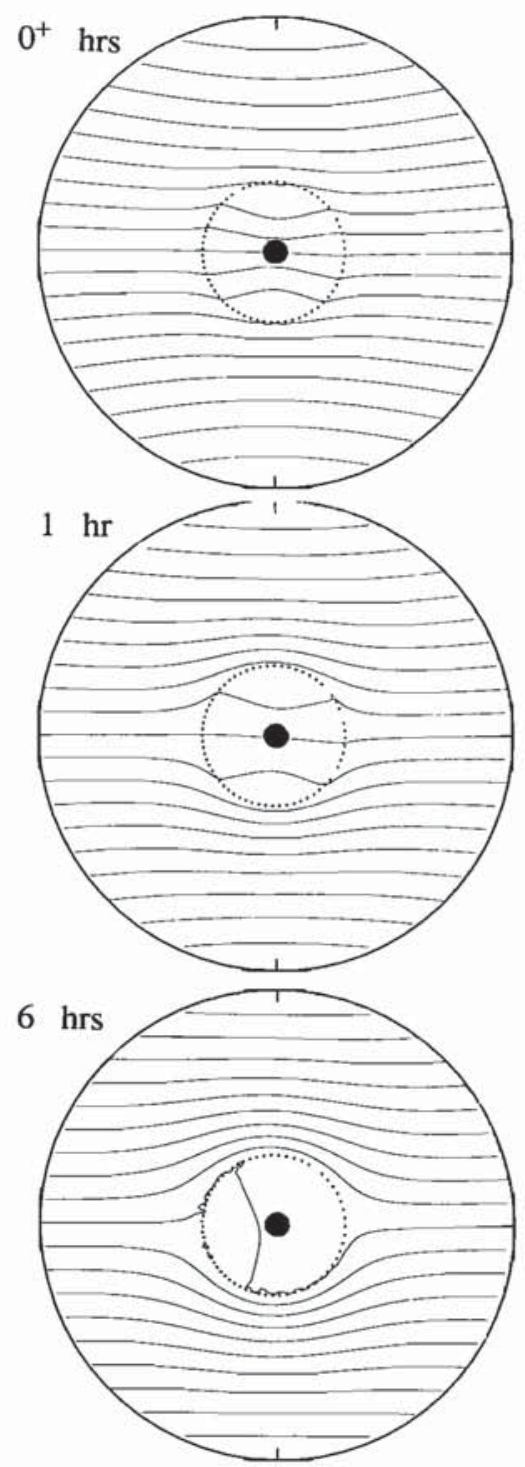

Fig. 11. Time dependent solution for the dipole model, starting from the steady state solution of Figure 10, shown immediately, 1 hour, and 6 hours after the cross-polar potential was increased from 40 to $100 \mathrm{kV}$. 

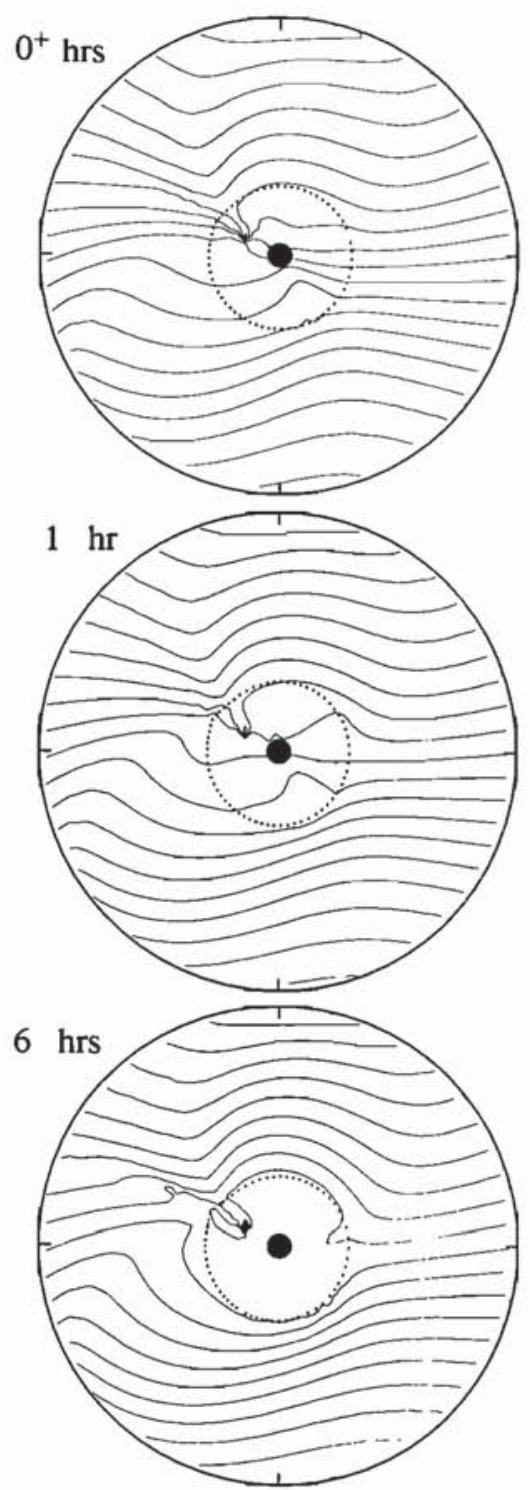

Fig. 12. Same as Figure 11 for the $Q_{3}$ model.

\section{COMPARISON WITH THE DATA}

The observations made by the Voyager 2 plasma science (PLS) experiment have been described by Bridge et al. [1986], McNutt et al. [1987], Selesnick and McNutt [1987] and Sittler et al. [1987]. For comparison with the results obtained here, the clearest presentation of the data is in the form of the energy-time spectrograms presented by Bridge et al. [1986], Selesnick and McNutt [1987] and Sittler et al. [1987]. A sketch of the important features (to the present discussion) of these spectrograms is shown in Figure 14. The top panel is for ions and the bottom panel for electrons. The vertical axis is a logarithmic energy-per-charge scale from $10 \mathrm{~V}$ to $6 \mathrm{kV}$ and the horizontal axis is time. The shaded regions show where plasma was detected. The cross-hatched region indicates higher intensity. The Voyager 2 trajectory plot from Selesnick and McNutt [1987] is reproduced in Figure 15. Here the location of the spacecraft has been projected along magnetic field lines into the equatorial plane. In this case the OTD field model was used. At the radial distances reached by Voyager 2 both the $Q_{3}$ and octupole models are well represented by the OTD model, so that Figure 15 is useful for displaying the location of the features in the data. The shaded blocks along the trajectory correspond roughly to the shaded regions in Figure 14. The circles of constant $L$ again refer to the OTD model. The closest approach to Uranus in OTD $L$ was 4.6 and is marked by $C . A$. in Figure 14 .

In general, it is extremely difficult to interpret features, such as those in Figure 14, from one spacecraft passage through the system, because of the inability to distinguish between spatial and temporal variations. Typically, for Earth orbiting satellites, a statistical study of many such data sets is needed [e.g., Mauk and Meng, 1983b, Fairfield and Vinas, 1984]. Also, the convection model described above is a simple one which neglects several effects such as gradients in the ionospheric conductivity and particle precipitation into the ionosphere. However, because of the unique nature of the data, we shall proceed keeping these statements in mind.

The most striking features of the data represented in Figure 14 are the sharp boundaries across the entire energy range centered roughly on the closest approach to the planet. These features have been interpreted by McNutt et al. [1987] and Selesnick and

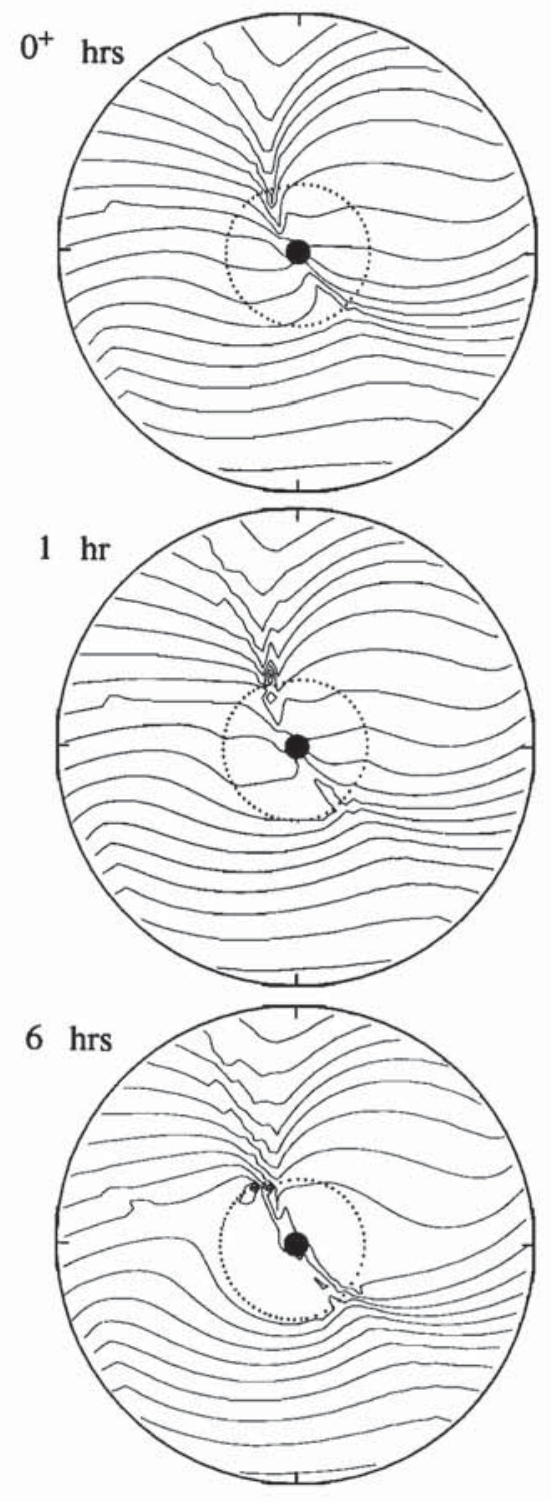

Fig. 13. Same as Figure 11 for the octupole model. 

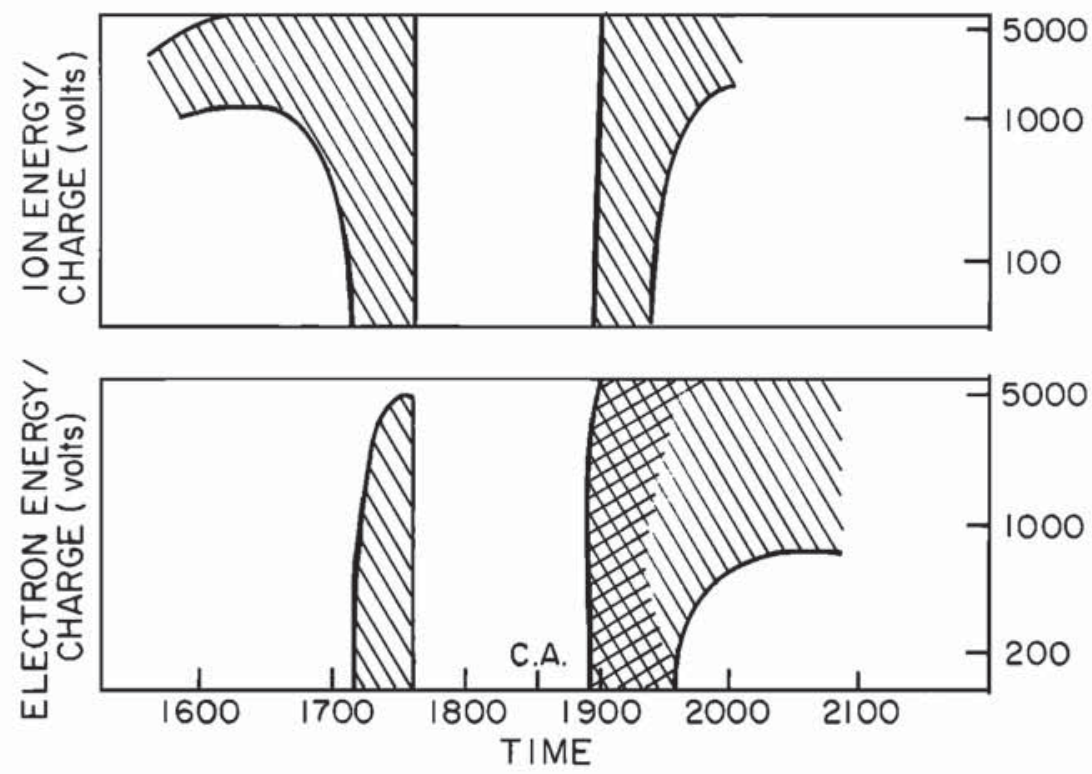

Fig. 14. Sketch of Voyager 2 plasma science experiment (PLS) energy-time spectrogram from the inner Uranian magnetosphere. The top panel is for ions and the bottom panel is for electrons. The shaded regions indicate where significant fluxes were observed.

McNutt [1987] as the innermost penetration of the sunward magnetospheric convection, similar to the Alfven layer, or forbidden zone, model for the plasma sheet inner edge in the Earth's magnetosphere [e.g., Kivelson et al., 1980]. To explain the sharpness of the boundaries at all energies, strong shielding of the external field, as depicted in Figure 10, is required. Here we only point out that such shielding is possible in the nondipolar magnetic field models.
The parameters used in the simulations of Figures 10 to 13 were chosen from the best available estimates. The energy near the inner edge of $5 \mathrm{keV}$ is near the upper limit of the PLS energy range for protons and the measured densities [Selesnick and McNutt, 1987] were sufficient for these particles to have provided the dominant contribution, of those observed, to the gradient/curvature drift currents. The observed value of $\eta \approx 10^{13} \mathrm{G}^{-1} \mathrm{~cm}^{-2}$ [Selesnick and McNutt, 1987] was combined

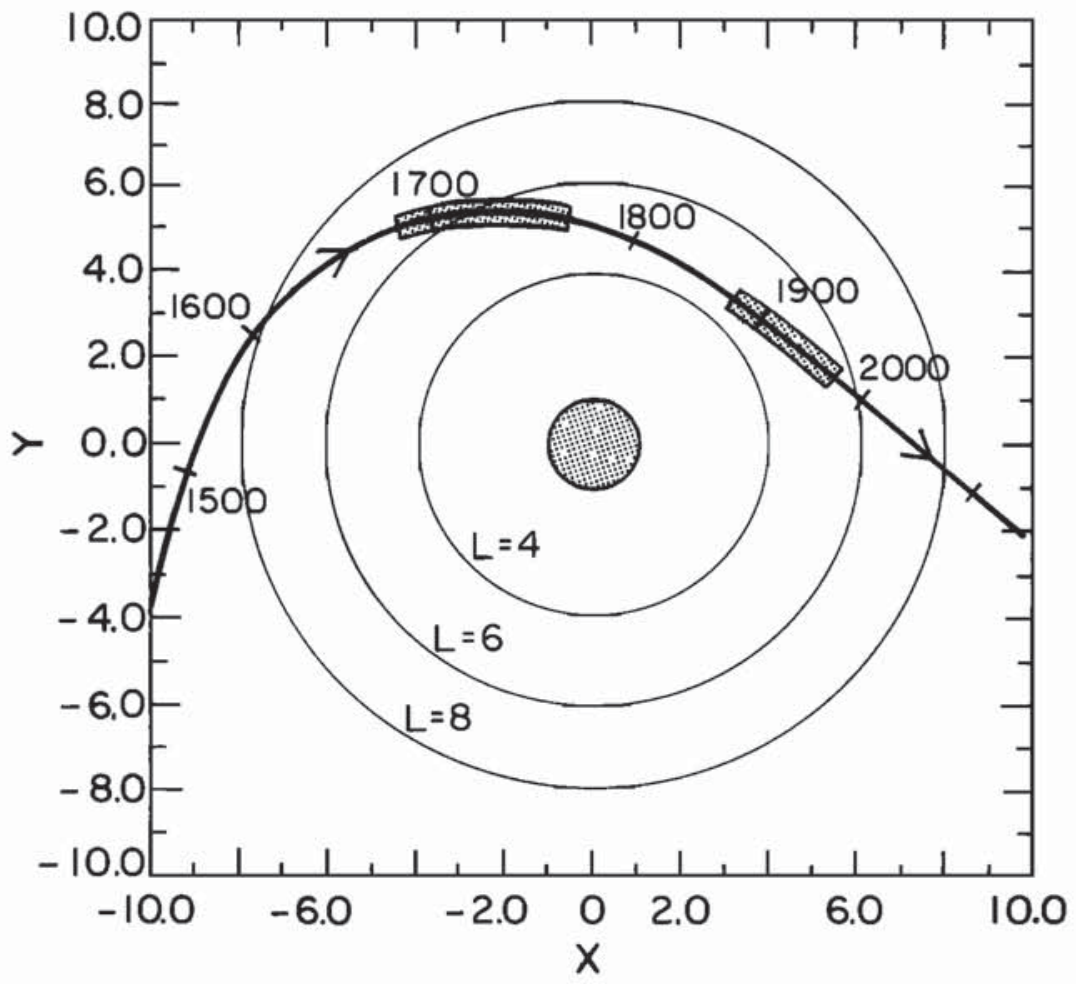

Fig. 15. Trajectory plot of Voyager 2 projected along OTD magnetic field lines into the equatorial plane. The tick marks along the trajectory show spacecraft event time (SCET) for comparison with Figure 14 and the shaded blocks correspond roughly to the shaded regions in Figure 14 where plasma was observed. 


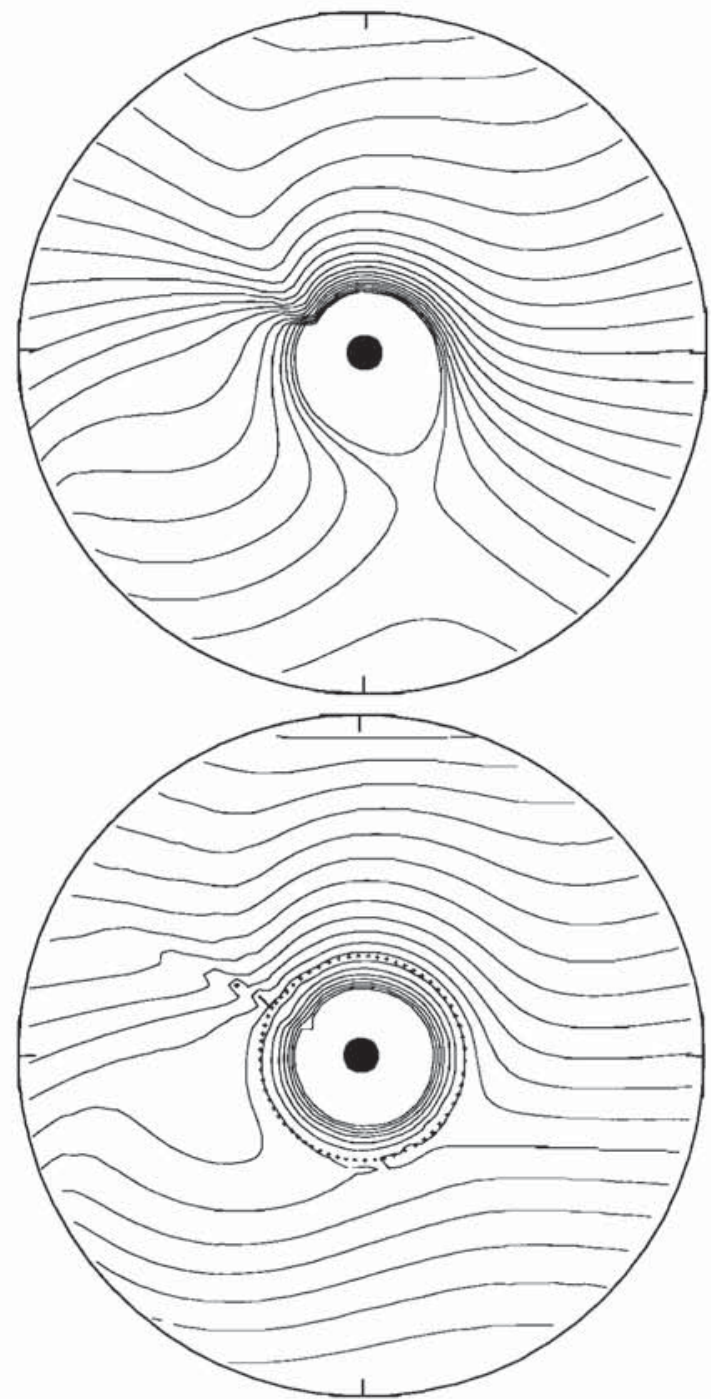

Fig. 16. Contours of total energy, $E_{k}+q \Phi$, for the $\mathrm{Q}_{3}$ model with $\lambda=2 \mathrm{MeV}\left(R_{U} / G\right)^{2 / 3}$. The top panel includes only the external field and the bottom panel includes both the external and steady-state self-consistent fields.

with $\Sigma_{P}=4$ mho to give $\eta q / \Sigma_{P}=40$. However, the value of $\Sigma_{P}$ is highly uncertain (see discussion by Selesnick and McNutt [1987]). The polar cap potential, $\Phi_{p}$, of 20 to $50 \mathrm{kV}$ was chosen to roughly agree with the various estimates of the time for plasma to convect through the magnetosphere in the range of about 1 to 10 days [Bridge et al., 1986; McNutt et al., 1987; Sittler et al., 1987].

To see how shielding can produce the sharp boundaries in the plasma data, contours of constant total energy, $E_{k}+q \Phi$, for particles with the same value of $\lambda$ as those included in the simulations, are plotted in Figure 16 using the $Q_{3}$ model. The top panel includes only the external field and the bottom panel includes both the external and steady state self-consistent fields. The total energy contours are streamlines of the bounce-averaged gradient/curvature plus $\mathbf{E} \times \mathbf{B}$ drift velocity, $\mathbf{v}_{\boldsymbol{\phi}}$ according to (4). The figure shows that the steady state inner edge becomes the outer limit of trapped orbits which are not accessible to convection from the magnetotail. For strong shielding this is true over a range of energies.

To maintain a sharp inner edge with the shielding mechanism, a quasi-steady state situation must exist in the inner magnetosphere, otherwise low energy plasma could cross the inner edge during periods when shielding is not strong, such as those depicted by the middle panels of Figures 11 to 13 . However, the orientation of the Uranian rotation axis implies that the angle between the planetary and interplanetary magnetic field changes with the rotation period. Several authors [Hill, 1986; McNutt et al., 1987; Sittler et al., 1987] have pointed out that the magnitude of the external field may oscillate as a result and possibly be accompanied by periodic substorm initiation. For the parameters used in the calculations of Figures 11,12 and 13 the time scale for reinstatement of strong shielding is not very short compared with the planetary rotation period of 17.24 hours derived by Desch et al. [1986]. A similar conclusion was reached by Sittler et al. [1987] using the analytic estimates of shielding time. Since $\eta$ and $\lambda$ are fairly well determined from the data as described above, the ionospheric Pedersen conductivity may have to be smaller than the value used if the shielding time is to become short compared to a rotation period. Given our present knowledge of the Uranian ionosphere this is certainly a possibility.

An alternative explanation for the sharpness of the boundary is evident from the potential contours of the external field in the $Q_{3}$ model, shown in Figures 6 and 8. Figure 15 shows that the boundaries were observed on the "dawn" (by analogy with the Earth) side of the planet. The potential contours in this region curve around the planet so that they are almost parallel to the inner edge, which is assumed to be roughly circular. Therefore even when the shielding is not strong, the plasma flow does not cross the boundary which can therefore stay sharp. Such a situation does not hold for the octupole model and we see from Figure 13 that the shielding is not effectively reinstated in the region where the contours show a strong flow across the boundary.

The location of the inner edge in the simulations of Figures 10 to 13 was chosen to agree with the Voyager 2 observations. However, the observed inner edge location at Uranus is much closer to the planet, relative to the size of the magnetosphere, than is typically observed at Earth. Vasyliunas [1972] showed that a large range of locations can be consistent with a given set of model parameters. Jaggi and Wolf [1973], Southwood [1977] and Siscoe [1982] derived formulae for the location that would be reached by the inner edge (in the limit of strong shielding) assuming it started from outside the range of consistent locations. McNutt et al. [1987] used this result (see also Selesnick and McNutt [1987]) to derive an approximate value for ionospheric Pedersen conductivity from the observed plasma parameters and location of the inner edge. The inner edge should form roughly at the radius where gradient drift speed becomes significant relative to the $\mathbf{E} \times \mathbf{B}$ drift speed. For the range of parameters discussed above this condition is roughly consistent with the plasma observed in the PLS energy range (the gradient/curvature drift period for $5 \mathrm{keV}$ protons at $L=6$ is about 12 days). However, a significant hot plasma population was detected by the Voyager 2 low energy charged particle (LECP) experiment [Krimigis et al., 1986; Mauk et al., 1987] simultaneously with the PLS observations. The PLS and LECP plasmas had comparable pressure (and therefore comparable values of $\eta \lambda$ ), but the LECP plasma did not show any pressure gradients comparable to the PLS gradients at the inner edge, indicating that it did not take part in the shielding. In fact, all of the LECP particles, which were observed at energies above $22 \mathrm{keV}$, would have been on trapped orbits for $\Phi_{p}<70 \mathrm{kV}$ (this value comes from setting the gradient and $\mathbf{E} \times \mathbf{B}$ drift speeds equal at a radius which maps to the edge of 


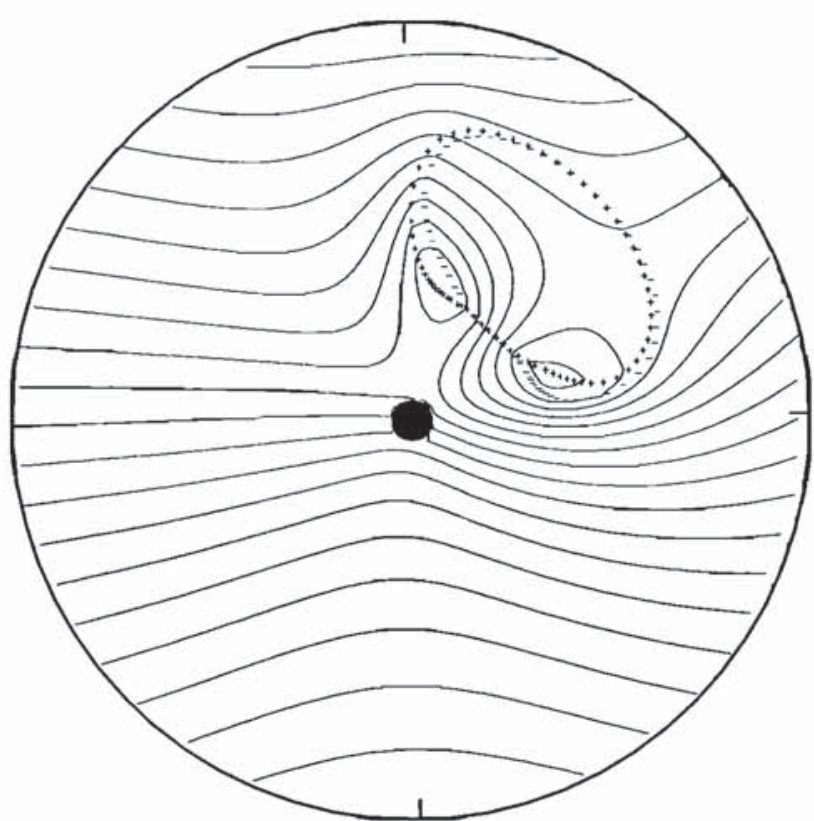

Fig. 17. Potential in the dipole magnetic field model due to a plasma cloud, which started as a circle with coincident proton (plus) and electron (minus) boundaries, after evolving for 4 hours.

the polar cap). If a significant plasma population exists at energies between those of the PLS and LECP observations, as one would expect by simple interpolation, then it is difficult to account for the location of the inner edge so close to the planet. In the "standard" model of magnetospheric convection presented here the hot particles which exist on closed orbits outside the inner edge do not play a role, but recent work (see review by Mauk and Zanetti [1987]) has suggested that they are actually important in current closure and, given the presence of the hot plasma at Uranus, the application of the standard model awaits full justification.

If the sharp boundaries do indeed represent an inner edge of the plasma sheet, then one would expect the plasma flux tube content, $\eta$, which is conserved by the flux tube drift, to be roughly constant outside the inner edge. Selesnick and McNutt [1987] have shown that this is actually not the case, and that $\eta$ decreases substantially outside the shaded regions in Figures 14 and 15. From Figures 14 and 15 we see that on the inbound portion of the Voyager 2 trajectory, the increase in $\eta$ began with the highest energy ions, was followed by the low-energy ions, then by low-energy electrons and finally high-energy electrons. Sittler et al. [1987] have suggested, based on a comparison with similar features observed in the Earth's magnetosphere, that this dispersion in arrival times is the result of a substorm injection followed by guiding center drifts. Such an effect would be similar to the injection boundary model which has been developed to explain features seen in plasma data obtained in the Earth's magnetosphere [McIlwain, 1974; Mauk and Meng, 1983a]. Although it may be successful in explaining the dispersive boundaries, the injection boundary model would seem to have difficulty with the sharp, dispersionless boundaries for which the quasi-steady state shielding model described above seems to have some success. Dispersionless features can be explained in the context of the injection boundary model by postulating that the injection took place with the spacecraft already in the vicinity of the injection site. However, since a dispersive feature was seen before the dispersionless feature on the inbound leg, the injection must have taken place before the spacecraft arrived, to allow time for the guiding center drifts to separate the protons and electrons.

The role of plasma injection, combined with a self-consistent field, can be illustrated with the convection model developed in this paper. The injection is postulated to bring all plasma populations to the same initial configuration. In the usual injection boundary model, the initial configuration is a double spiral centered roughly on local midnight [Mauk and Meng, 1983a]. Here we choose to start with a circular plasma cloud in the post-midnight sector, because this configuration gives a clearer illustration of the effects of gradient and curvature drifts. The results, after running for a simulated 4 hours in the dipole field model, are shown in Figure 17. Here the initial boundary consisted of both proton (indicated by plus signs) and electron (indicated by minus signs) components, each with $\eta q / \Sigma_{p}=2$ and $\lambda=2 \mathrm{MeV}\left(R_{U} / \mathrm{G}\right)^{2 / 3}$, and with $\Phi_{p}=20 \mathrm{keV}$. The self-consistent field of both components was included. The initial circle was centered a distance of $7 R_{U}$ along both the $x$ and $y$ axes with a radius of $6 R_{U}$. No other plasma boundaries were included in the simulation. The protons have clearly drifted ahead of the electrons due to the opposite directions of their gradient and curvature drifts. Also, the self-consistent field has caused a section of the cloud on the planetward side to maintain the coincidence between the two boundaries, which would not occur for drifts in the external field only. Thus it may be possible to explain the existence of both the dispersive and dispersionless boundaries with the combined effects of substorm injection and the self-consistent field, although a detailed matching of such a theory with the observations is beyond the scope of the present model.

The region of high-intensity electrons on the outbound leg of the Voyager 2 trajectory, shown in Figure 14 by the cross-hatched region, may be the result of temporal variations or particle losses leading to azimuthal asymmetries. Neither of these effects are included in the model presented here. However, azimuthal asymmetries do result from the $\mathrm{Q}_{3}$ and octupole models which may enhance the effect. For example, the octupole model produces a distinct difference in convection speed between the pre- and postmidnight sectors, as seen in Figures 9, 10 and 13. The regions with smaller convection speeds would be more susceptible to particle losses by precipitation into the ionosphere or charge exchange with neutral hydrogen, and this may be responsible for the reduced electron intensities on the inbound leg. Assuming strong pitch angle scattering the loss lifetime into the ionosphere can be estimated from $\tau=B_{i} V\left(2 m / E_{k}\right)^{1 / 2}[W o l f$, 1983] where $m$ is the particle mass. Near the inner edge the loss lifetimes are about 1 hour for electrons and 40 hours for ions at 5 $\mathrm{keV}$. While these lower limits on the lifetimes indicate that losses are significant, it is not likely that strong pitch angle scattering is reached [Sittler et al., 1987] (note that this does not effect our initial assumption of an isotropic plasma because the model is not sensitive to small anisotropies). If losses are significant, which is indicated at least in the Earth's magnetosphere, then the convection model must be modified to include their effects [Southwood and Wolf, 1978; Caudal, 1987].

\section{SUMMARY}

Our goal has been to study the aspects of magnetospheric convection which are unique to Uranus due to its unusual planetary magnetic field. A method for determining the convection electric field using simple analytic approximations under the assumption of uniform ionospheric conductivity has been described. Magnetic field models including quadrupole and 
octupole moments have been used to determine the shape of the polar caps and the mapping of the electric field and parallel currents between ionosphere and magnetosphere. The asymmetry in the magnetic field models between the northern and southern hemispheres leads to the inclusion of currents between the hemispheres in order to satisfy the assumption of equipotential magnetic field lines.

The results show that the quadrupole moment of the Uranian magnetic field strongly influences magnetospheric convection, and that a significant octupole moment will further alter the flow pattern. The basic flow remains sunward in the inner magnetosphere. The total current which flows along field lines between the two hemispheres due to the asymmetry of the magnetic field is comparable in magnitude to that of the region 1 current system, although the net current between the hemispheres is small. As a result of this current the northern magnetic hemisphere has a dominant influence in the determination of the convection electric field. Time dependent calculations including a self-consistent electric field have been made, and show that ring current shielding of the electric field can be important and may have formed the dominant features in the plasma observations made by Voyager 2 . However, uncertainties in the model parameters make it difficult to determine whether the shielding time is short enough to maintain the plasma features under time variations due to the planetary rotation. The effectiveness of the shielding can also be influenced by the magnetic field model. Other features in the data are characteristic of substorm injection, and the model has been used to show that a combination of plasma injection and electric field shielding may be applicable.

Acknowledgments. The author thanks R. L. McNutt, Jr., J. D. Richardson, and J. W. Belcher for helpful discussions and comments on the manuscript, J. E. P. Connemey for providing the magnetic field mode parameters prior to publication, and both referees for suggestions which improved the paper. This work was supported under NASA contract 957781 to the Jet Propulsion Laboratory and NASA grant NAGW-1209.

The editor thanks B. H. Mauk and another referee for their assistance in evaluating this paper.

\section{REFERENCES}

Behannon, K. W., R. P. Lepping, N. F. Ness, E. C. Sittler, Jr., B. H. Mauk, S. M. Krimigis, and R. L. McNutt, Jr., The magnetotail of Uranus, $J$. Geophys. Res., 92, 15,354-15,366, 1987.

Bridge, H. S., et al., Plasma observations near Uranus: Initial results from Voyager 2, Science, 233, 89-93, 1986.

Caudal, G., Field-aligned currents from EISCAT radar observations and implications concerning the mechanism that produces region 2 currents, J. Geophys. Res., 92, 6000-6012, 1987.

Connemey, J. E. P., M. H. Acuna, and N. F. Ness, The magnetic fleld of Uranus, J. Geophys. Res., 92, 15,329-15,336, 1987.

Desch, M. D., J. E. P. Connemey, and M. L. Kaiser, The rotation period of Uranus, Nature, 322, 42-43, 1986.

Dolginov, Sh. Sh., The magnetic field of the planet Uranus: predictions, measurements and interpretation, Cosmic Res., 25, 244-255, 1986.

Fairfield, D. H. and A. F. Vinas, The inner edge of the plasma sheet and the diffuse aurora, J. Geophys. Res., 89, 841-854, 1984

Fejer, J. A., Semidiurnal currents and electron drifts in the ionosphere, $J$. Atmos. Terr. Phys., 4, 184-203, 1953.

Fejer, J. A., Theory of geomagnetic daily disturbance variations, J. Geophys. Res., 69, 123-137, 1964.

Harel, M., R. A. Wolf, P. H. Reiff, R. W. Spiro, W. J. Burke, F. J. Rich, and M. Smiddy, Quantitative study of a magnetospheric substorm, 1, Model logic and overview, J. Geophys. Res., 86, 2217-2241, 1981.

Hill, T. W., The magnetosphere of Uranus: A resonantly driven oscillator?, Eos Trans. AGU, 67, 341, 1986.

Jaggi, R. K. and R. A. Wolf, Self-consistent calculation of the motion of a sheet of ions in the magnetosphere, J. Geophys. Res., 78, 2852-2866, 1973.

Kivelson, M. G., S. M. Kaye, and D. J. Southwood, The physics of plasma injection invents, in Dynamics of the Magnetosphere, edited by S. I. Akasofu, pp. 385-405, D. Reidel, Hingham, Mass., 1980.
Krimigis, S. M., T. P. Armstrong, W. I. Axford, A. F. Cheng, G. Gloeckler, D. C. Hamilton, D. C. Keath, L. J. Lanzerotti, and B. H. Mauk, The magnetosphere of Uranus: Hot plasma and radiation environment, Science, 233, 97-102, 1986.

Mauk, B. H., S. M. Krimigis, E. P. Keath, A. F. Cheng, T. P. Armstrong, L. J. Lanzerotti, G. Gloeckler, and D. C. Hamilton, The hot plasma and radiation environment of the Uranian magnetosphere, J. Geophys. Res. $92,15,283-15,308,1987$

Mauk, B. H. and C.-I. Meng, Characterization of geostationary particle signatures based on the injection boundary model, J. Geophys. Res., 88 , 3055-3071, 1983a.

Mauk, B. H. and C.-I. Meng, Dynamical injections as the source of near geostationary quiet time particle spatial boundaries, J. Geophys. Res., $88,10,011-10,024,1983 b$.

Mauk, B. H. and L. J. Zanetti, Magnetospheric electric fields and currents, Rev. Geophys., 25, 541-554, 1987.

McIlwain, C. E., Substorm injection boundaries, in Magnetospheric Physics, edited by B. M. McCormac, p. 143, D. Reidel, Hingham, Mass., 1974.

Mcllwain, C. E., A $K_{p}$ dependent equatorial electric field model, $A d v$. Space Res., 6, 187-197, 1986.

McNutt, R. L. Jr.n, R. S. Selesnick, and J. D. Richardson, Low-energy plasma observations in the magnetosphere of Uranus, J. Geophys. Res., 92, 4399-4410, 1987.

Menke, W., Geophysical Data Analysis: Discrete Inverse Theory, Academic, San Diego, Calif., 1984.

Morse, P. M. and H. Feshbach, Methods of Theoretical Physics, McGraw-Hill, New York, 1953.

Ness, N. F., M. H. Acuna, K. W. Behannon, L. F. Burlaga, J. E. P. Connerney, R. P. Lepping, and F. M. Neubauer, Magnetic fields at Uranus, Science, 233, 85-89, 1986.

Saff, E. B. and A. D. Snider, Fundamentals of Complex Analysis for Mathematics, Science and Engineering, Prentice-Hall, Englewood Cliffs, N. J., 1976.

Selesnick, R. S., Magnetospheric convection at Uranus, in $S P I$. Conference Proceedings and Reprint Series, Vol. 6, edited by T. Chang, Scientific Publishers, Cambridge, Mass., 1987.

Selesnick, R. S. and R. L. McNutt, Jr., Voyager 2 plasma ion observations in the magnetosphere of Uranus, J. Geophys. Res., 92, 15,249-15,262, 1987.

Selesnick, R. S. and J. D. Richardson, Plasmasphere formation in arbitrarily oriented magnetospheres, Geophys. Res. Lett., 13, 624-627, 1986.

Senior, C. and M. Blanc, On the control of magnetospheric convection by the spatial distribution of ionospheric conductivities, J. Geophys. Res., $89,261-284,1984$.

Siscoe, G. L., Energy coupling between regions 1 and 2 Birkland current systems, J. Geophys. Res., 87, 5124-5130, 1982.

Sittler, E. C. Jr., K. W. Ogilvie, and R. S. Selesnick, Survey of electrons in the Uranian magnetosphere: Voyager 2 observations, J. Geophys. Res., $92,15,263-15,281,1987$.

Southwood, D. J., The role of hot plasma in magnetospheric convection, $J$. Geophys. Res., 82, 5512-5520, 1977.

Southwood, D. J. and R. A. Wolf, An assessment of the role of precipitation in magnetospheric convection, J. Geophys. Res., 83, 5227-5232, 1978.

Stern, D. P., Large-scale electric fields in the Earth's magnetosphere, Rev. Geophys. , 15, 156-194, 1977.

Vasyliunas, V. M., Mathematical models of magnetospheric convection and its coupling to the ionosphere, in Particles and Fields in the Magnetosphere, edited by B. M. McCormac, pp. 60-71, D. Reidel, Hingham, Mass., 1970.

Vasyliunas, V. M., The interrelationships of magnetospheric processes, in Earth's Magnetospheric Processes, edited by B. M. McCormac, pp. 29-38, D. Reidel, Hingham, Mass., 1972.

Vasyliunas, V. M., The convection-dominated magnetosphere of Uranus, Geophys. Res. Lett., 13, 621-623, 1986.

Volland, H., A semiempirical model of large-scale magnetospheric electric fields, J. Geophys. Res., 78, 171-180, 1973.

Wolf, R. A., The quasi-static (slow-flow) region of the magnetosphere, in Solar-Terrestrial Physics, edited by R. L. Carovillano, pp. 303-368, D. Reidel, Hingham, Mass., 1983.

R. S. Selesnick, 220-47 Califomia Institute of Technology, Pasadena, CA 91125

(Received February 23, 1988;

revised May 25, 1988;

accepted May 26, 1988.) 